\title{
WELL-POSEDNESS, ROBUSTNESS, AND STABILITY ANALYSIS OF A SET-VALUED CONTROLLER FOR LAGRANGIAN SYSTEMS
}

\author{
SAMIR ADLY ${ }^{\dagger}$, BERNARD BROGLIATO ${ }^{\ddagger}$, AND BA KHIET LE ${ }^{\dagger}$
}

\begin{abstract}
This paper deals with the analysis of a class of nonsmooth robust controllers for Lagrangian systems with nontrivial mass matrix. First the existence and uniqueness of solutions are analyzed, then the Lyapunov stability, the Krasovskii-LaSalle invariance principle, and finite-time convergence properties are studied.
\end{abstract}

Key words. Lagrangian systems, set-valued systems, Lyapunov stability, Krasovskii-LaSalle invariance principle, finite-time convergence, robust control

1. Introduction. The aim of this paper is to study a class of nonlinear Lagrange dynamical systems with a multivalued controller of the form

$$
M(q(t)) \ddot{q}(t)+C(q(t), \dot{q}(t)) \dot{q}(t)+\nabla \mathcal{V}(q(t))+F(t, q(t), \dot{q}(t)) \in-\partial \Phi(\dot{q}(t))
$$

for a.e. $t \geq t_{0}$, where $t_{0} \in \mathbb{R}$ is fixed, $\Phi: \operatorname{dom}(\Phi)=\mathbb{R}^{n} \rightarrow \mathbb{R}$ is a convex function, $\mathcal{V} \in \mathcal{C}^{1}\left(\mathbb{R}^{n} ; \mathbb{R}\right)$ with its gradient $\nabla \mathcal{V}(\cdot)$, the matrices $M(q), C(q, \dot{q}) \in \mathbb{R}^{n \times n}$, and $\partial \Phi(\cdot)$ stands for the convex subdifferential of $\Phi(\cdot)$. Motivated by control applications, we consider in this work the case where the set-valued function in (1.1) depends on the velocity uniquely. Other works $[35,39]$ have focused on the case when the setvalued part is the normal cone to a subset of $\mathbb{R}^{n}$, which depends on the position uniquely. This yields different types of dynamics with unilateral constraints and discontinuities in the velocity. The vector $q$ represents the generalized coordinates, $n$ is the number of degrees of freedom, $M(q)$ is the inertia matrix, $C(q, \dot{q})$ is the centripetal-Coriolis matrix. The function $F(t, q(t), \dot{q}(t))$ represents a perturbation force which is usually bounded by a constant. The terms $\nabla \mathcal{V}(q(t))$ and $-\partial \Phi(\dot{q}(t))$ may represent a control input $u(q, \dot{q})=-\nabla \mathcal{V}(q)-\partial \Phi(\dot{q})$ applied to stabilize the system (in finite time) at some fixed point. The advantage of such controllers is that they are intrinsically robust since the a priori knowledge of the system's parameters (like the inertial parameters) is not necessary for stabilization, and an upper bound of the disturbance is sufficient to reject it. In addition the closed-loop system trajectories attain the equilibrium point in finite time. The robust discontinuous controller is an important area of research in systems and control $[5,6,10,21,28,33,43,44,46]$, where the so-called sliding mode inputs are analyzed. Robust controllers guaranteeing finite-time stability are applied, among many other applications, to mechanical and electromechanical systems [19, 25, 26, 40, 45, 47]. Finite-time convergence properties are also studied in the mathematical and control literature [1, 12, 18, 20, 31, 32, 34], as well as stability and invariance properties of nonsmooth systems and differential inclusions $[8,9,15,16,24,29,30,33,38]$. Many of these works are based on the

\footnotetext{
†XLIM UMR-CNRS 7252, Université de Limoges, 87060 Limoges, France (samir.adly@unilim.fr, ba-khiet.le@xlim.fr).

${ }^{\ddagger}$ INRIA Grenoble Rhône-Alpes, 38334 Grenoble, France (bernard.brogliato@inria.fr).
} 
so-called Filippov's differential inclusions and solutions [20]. In other works, maximal monotonicity is the central property, as in $[2,3,13,14,15,16]$. In this paper the closedloop dynamics in (1.1) is analyzed. The existence and uniqueness of solutions are first carefully studied, and in a second stage stability properties are examined. The tools are those of convex analysis, which are combined with the dynamical properties of Lagrangian systems. Compared to some previous works dealing with similar dynamics [2], in this paper we consider a nonconstant mass matrix $M(q)$ in (1.1). As we shall see, this is not a trivial matter because it may destroy the monotonicity of the operator which appears in the first order formulation of (1.1), i.e., $z=\left(z_{1}, z_{2}\right) \mapsto$ $M^{-1}\left(z_{1}\right) \partial \Phi\left(z_{2}\right)$. In other words, the change of variables used in $[2,3,14,15,16]$ to recast some set-valued systems into maximal monotone differential inclusions, and which uses the chain rule of convex analysis [42, Theorem 4.2.1], no longer works for nontrivial mass matrices. Since uniqueness may not hold for such systems, we propose a stability analysis that also encompasses nonunique solutions.

The analysis in this paper may also be seen as a first step for the study of the digital implementation of such discontinuous controllers, using implicit Euler of zeroorder-hold discretizations along the lines of $[3,4]$. Such digital implementations allow suppression of so-called numerical chattering $[22,23]$, which is a highly undesirable effect in practice, especially in mechanical structures where one wants to decrease as much as possible the vibrations. They also permit keeping in discrete time the finite-time convergence property, i.e., the attractive surfaces are attained after a finite number of steps.

The paper is organized as follows. In section 2 some useful results and definitions from convex analysis are recalled. Section 3 is dedicated to the properties of Lagrangian dynamics and characterizes the disturbance as well as an important property of a family of functions $\Phi(\cdot)$. In section 4 the existence of solutions issue is tackled through the study of Moreau-Yosida approximations of $\Phi(\cdot)$. In section 5 the conditions under which uniqueness of solutions hold are examined. Lyapunov stability and finite-time convergence are studied in sections 6 and 7, respectively; a stability framework that allows for nonunique solutions, an well as an extension of the Krasovskii-LaSalle invariance principle, are proposed. The sets of switching instants where the velocity attains zero value are studied, hence refining the characterization of the solutions; an estimation of the settling time is provided. Conclusions end the paper in section 8.

2. Notations and mathematical background. Denote by $\langle\cdot, \cdot\rangle,\|\cdot\|$ the scalar product and the corresponding norm (the euclidean norm), by $\|\cdot\|_{1}$ the 1 -norm in $\mathbb{R}^{n}$. We use the notation $\|\cdot\|_{m}$ for the induced matrix norm. Denote by $I$ the identity operator, $I_{n}$ the identity matrix of size $n, \mathbb{B}_{h}$ the closed ball of radius $h$ centered at 0 , and $\mathbb{B}_{h}(s)$ the closed ball of radius $h$ centered at $s$. For a set $K$, its boundary is denoted by $\operatorname{bd}(K)$ and the distance from a point $s$ to $K$ is denoted by $d(s, K)$.

Definition 2.1. Assume a square matrix $M \in \mathbb{R}^{n \times n} . M$ is called positive definite if for every $x \in \mathbb{R}^{n} \backslash\{0\}$, we have

$$
\langle M x, x\rangle>0 .
$$

The induced norm of $M$ is defined by

$$
\|M\|_{m}:=\sup _{x \in \mathbb{R}^{n}, x \neq 0} \frac{\|M x\|}{\|x\|}=\sup _{x \in \mathbb{R}^{n},\|x\|=1}\|M x\| .
$$


Definition 2.2. A continuously differentiable function $V: \mathbb{R}^{n} \rightarrow \mathbb{R}$ is called (1) radially unbounded if

$$
V(x) \rightarrow \infty \text { as }\|x\| \rightarrow \infty
$$

(2) locally positive definite if there exists $\varepsilon>0$ such that

$$
V(0)=0 \text { and } V(x)>0 \forall x \in \mathbb{B}_{\varepsilon} .
$$

Proposition 2.3. If a $\mathcal{C}^{1}$ function $V(\cdot)$ is locally positive definite, then there exist $\varepsilon>0$ and a strictly increasing function $\rho \in \mathcal{C}\left(\mathbb{R}^{+} ; \mathbb{R}\right)$ with $\rho(0)=0$ such that

$$
V(x) \geq \rho(\|x\|) \forall x \in \mathbb{B}_{\varepsilon} .
$$

The following material on maximal monotone operators, convex functions, and upper semicontinuous functions can be found in $[8,11,13,36]$. Finally, a version of Gronwall's inequality is recalled. Let $X$ be a Hilbert space.

Definition 2.4. A set-valued map $A(\cdot)$ from $X$ into the subsets of its dual $X^{*} \equiv X$ is said to be a monotone operator provided

$$
\left\langle x_{2}-x_{1}, y_{2}-y_{1}\right\rangle \geq 0 \quad \forall x_{1}, x_{2} \in X \text { and } y_{1} \in A\left(x_{1}\right), y_{2} \in A\left(x_{2}\right) \text {. }
$$

The graph of $A(\cdot)$ is defined by

$$
G(A):=\{(x, y): y \in A(x)\}
$$

Definition 2.5. A monotone set-valued map $A(\cdot)$ is called maximal if there is no other monotone set-valued map $B(\cdot)$ such that graph of $A(\cdot)$ is contained strictly in graph of $B(\cdot)$.

Proposition 2.6. A set-valued map $A(\cdot)$ is maximal monotone if and only if the following statements are equivalent:

(a) For every $\left(x_{1}, y_{1}\right) \in G(A),\left\langle x_{2}-x_{1}, y_{2}-y_{1}\right\rangle \geq 0$.

(b) $y_{2} \in A\left(x_{2}\right)$.

THEOREM 2.7 (Yosida approximation $[8$, p. 144]). Let $A(\cdot)$ be maximal monotone and let $\lambda>0$. Then

(1) the resolvent of $A(\cdot)$ (of index $\lambda$ ) defined by $J_{\lambda}:=(I+\lambda A)^{-1}$ is a nonexpansive single valued map from $X$ to $X$;

(2) the Yosida approximation (of index $\lambda$ ) of $A(\cdot)$ defined by $A_{\lambda}:=\frac{1}{\lambda}\left(I-J_{\lambda}\right)$ satisfies

(i) for all $x \in X, A_{\lambda}(x) \in A\left(J_{\lambda} x\right)$,

(ii) $A_{\lambda}(\cdot)$ is Lipschitz with constant $\frac{1}{\lambda}$ and maximal monotone.

Definition 2.8. A set-valued map $A: X \rightrightarrows X$ is called hypomonotone provided that there exists a real $k>0$ such that for all $x_{1}, x_{2} \in X, y_{1} \in A\left(x_{1}\right), y_{2} \in A\left(x_{2}\right)$, we have

$$
\left\langle y_{1}-y_{2}, x_{1}-x_{2}\right\rangle \geq-k\left\|x_{1}-x_{2}\right\|^{2}
$$

The map $A(\cdot)$ is called locally hypomonotone if for each $x_{0} \in X, A(\cdot)$ is hypomonotone in a neighborhood of $x_{0}$.

Definition 2.9. Let $F: \mathbb{R}^{n} \rightrightarrows \mathbb{R}^{m}$ be a set-valued function. One says that $F(\cdot)$ is upper semicontinuous at $x_{0} \in \mathbb{R}^{n}$ if for any open neighborhood $\mathcal{N}$ containing $F\left(x_{0}\right)$ there exists an open neighborhood $\mathcal{M}$ of $x_{0}$ such that $F(\mathcal{M}) \subset \mathcal{N}$. 
Proposition 2.10 (see $[8$, p. 41$]$ ). Let $F_{1}: \mathbb{R}^{n} \rightrightarrows \mathbb{R}^{m}$ and $F_{2}: \mathbb{R}^{m} \rightrightarrows \mathbb{R}^{q}$ be two set-valued functions. Define $F_{2} \circ F_{1}: \mathbb{R}^{n} \rightrightarrows \mathbb{R}^{q}$ by

$$
\left(F_{2} \circ F_{1}\right)(x):=\bigcup_{y \in F_{1}(x)} F_{2}(y) .
$$

If $F_{1}(\cdot)$ and $F_{2}(\cdot)$ are upper semicontinuous, then $\left(F_{2} \circ F_{1}\right)(\cdot)$ is upper semicontinuous. In particular, $\left(F_{1}+F_{2}\right)(\cdot)$ is upper semicontinuous.

Proposition 2.11 (see [20, p. 66]). Let a set $D$ be closed and a set-valued function $F(\cdot)$ with closed values be bounded in a neighborhood of each point $p \in D$. Then the function $F(\cdot)$ is upper semicontinuous on $D$ if and only if its graph is closed.

Definition 2.12. Let $f(\cdot)$ be a convex function from $\mathbb{R}^{n}$ to $\mathbb{R}$. The subdifferential of $f(\cdot)$ at $x$ is defined by

$$
\partial f(x):=\left\{v \in \mathbb{R}^{n}:\langle v, y-x\rangle \leq f(y)-f(x) \forall y \in \mathbb{R}^{n}\right\} .
$$

Proposition 2.13 (see $[11,36]$ ). Let $f(\cdot)$ be a convex function from $\mathbb{R}^{n}$ to $\mathbb{R}$. Then

(1) $f(\cdot)$ is locally Lipschitz;

(2) $\partial f(\cdot)$ is maximal monotone and bounded on bounded sets;

(3) $\partial f(\cdot)$ is upper semicontinuous with nonempty, convex, and compact values.

Proposition 2.14 (Gronwall's inequality). Let $T>0$ be given and let $b \in$ $L^{1}\left(\left[t_{0}, t_{0}+T\right], \mathbb{R}\right)$. Let the absolutely continuous function $u:\left[t_{0}, t_{0}+T\right] \rightarrow \mathbb{R}$ satisfy

$$
\dot{u}(t) \leq b(t) u(t) \text {, a.e } t \in\left[t_{0}, t_{0}+T\right]
$$

Then

$$
u(t) \leq u\left(t_{0}\right) \exp \left(\int_{t_{0}}^{t} b(s) d s\right) \forall t \in\left[t_{0}, t_{0}+T\right] .
$$

Definition 2.15. Given $1 \leq k, p \leq \infty$. A Sobolev space $\mathcal{W}^{k, p}\left([0, T] ; \mathbb{R}^{n}\right)$ is defined by

$$
\begin{aligned}
& \mathcal{W}^{k, p}\left([0, T] ; \mathbb{R}^{n}\right) \\
& \quad:=\left\{u \in L^{p}\left([0, T] ; \mathbb{R}^{n}\right): u^{\prime}, \ldots, u^{(k)} \text { exist and belong to } L^{p}\left([0, T] ; \mathbb{R}^{n}\right)\right\},
\end{aligned}
$$

where the derivatives $u^{\prime}, \ldots, u^{(k)}$ are understood in the weak sense.

3. Properties of the Lagrangian dynamics. In this section, some fundamental assumptions are made and crucial properties of the Lagrange dynamics are recalled.

Assumption 3.1 (the inertia matrix).

$\left(\mathcal{M}_{1}\right) M(q)$ is symmetric for all $q \in \mathbb{R}^{n}$ and there exist $k_{1}>0, k_{2}>0$ such that

$$
k_{1} I_{n} \leq M(q) \leq k_{2} I_{n}
$$

i.e., for all $q, x \in \mathbb{R}^{n}$, we have $k_{1}\|x\|^{2} \leq\langle M(q) x, x\rangle \leq k_{2}\|x\|^{2}$.

$\left(\mathcal{M}_{2}\right)$ There exists $k_{3}>0$ such that for all $u, v, w \in \mathbb{R}^{n}$,

$$
\|M(u) w-M(v) w\| \leq k_{3}\|u-v\|\|w\|
$$

Assumption 3.2.

$\left(\mathcal{C}_{1}\right)$ There exists $k_{4}>0$ such that for all $u, v \in \mathbb{R}^{n}$,

$$
\|C(u, v)\|_{m} \leq k_{4}\|v\| \text {. }
$$


$\left(\mathcal{C}_{2}\right)$ For all $t \geq t_{0}$, we have

$$
\frac{d}{d t}(M(q(t)))=C(q(t), \dot{q}(t))+C(q(t), \dot{q}(t))^{T} .
$$

In particular Assumption $\left(\mathcal{C}_{2}\right)$ holds if $C(q, \dot{q})$ is defined from the so-called Christoffel's symbols.

Assumption 3.3.

$\left(\mathcal{H}_{\Phi}\right) \min _{x \in \mathbb{R}^{n}} \Phi(x)=\Phi(0)=0$.

$\left(\mathcal{H}_{C}\right)$ The function $h: \mathbb{R}^{n} \times \mathbb{R}^{n} \rightarrow \mathbb{R}^{n}$ defined by $h\left(x_{1}, x_{2}\right)=C\left(x_{1}, x_{2}\right) x_{2}$ is locally Lipschitz.

$\left(\mathcal{H}_{F}\right)$ The function $F\left(t, x_{1}, x_{2}\right)$ from $\mathbb{R} \times \mathbb{R}^{n} \times \mathbb{R}^{n} \rightarrow \mathbb{R}^{n}$ is continuous in $t$, uniformly locally Lipschitz in $x_{1}, x_{2}$ (i.e., the Lipschitz constant is independent of $t$ ) and bounded.

$\left(\mathcal{H}_{\Phi, F}\right)$ There exists $\lambda^{*}>0$ such that for all $t \geq 0, x, y \in \mathbb{R}^{n}$, and $0<\lambda \leq \lambda^{*}$ we have

$$
\left\langle\nabla \Phi_{\lambda}(y)+F(t, x, y), y\right\rangle \geq 0,
$$

where $\Phi_{\lambda}(\cdot)$ is the Moreau-Yosida approximation of $\Phi(\cdot)$ for each $\lambda>0$, i.e.,

$$
\Phi_{\lambda}(\cdot):=\inf _{z \in R^{n}}\left\{\Phi(z)+\frac{1}{2 \lambda}\|z-\cdot\|^{2}\right\} .
$$

$\left(\mathcal{H}_{\mathcal{V}}-\mathrm{i}\right) \mathcal{V}(\cdot)$ is $\mathcal{C}^{1}$ and $\nabla \mathcal{V}(\cdot)$ is Lipschitz continuous on bounded sets.

$\left(\mathcal{H}_{\mathcal{V}}-\mathrm{ii}\right) \mathcal{V}(\cdot)$ is bounded from below.

Remark 3.1. Assumptions $\left(\mathcal{M}_{1}\right),\left(\mathcal{M}_{2}\right),\left(\mathcal{C}_{1}\right),\left(\mathcal{C}_{2}\right),\left(\mathcal{H}_{C}\right)$ are used for the design of stabilizing controllers in robotics $[7,41]$. They are typically used, together with $\left(\mathcal{H}_{\mathcal{V}}-\mathrm{ii}\right)$, to prove the dissipativity of the Lagrange dynamics [17]. The function $F(\cdot)$ plays a role of perturbation force which is usually bounded by a constant. If the system is not subject to disturbances, i.e., $F(\cdot) \equiv 0$, the property $\left(\mathcal{H}_{\Phi, F}\right)$ of Assumption 3.3 naturally holds for all $\lambda^{*}>0$. This means that if the magnitude of perturbation force is small enough, this property holds for a large class of function $\Phi$.

The following lemma shows how to compute the Moreau-Yosida approximation of the euclidean and 1-norm functions. This result will be used in Lemma 3.2 to give some cases where the property $\left(\mathcal{H}_{\Phi, F}\right)$ is satisfied.

LEMMA 3.1. The following hold:

(i) If $\Phi(\cdot)=\|\cdot\|$, then $\Phi_{\lambda}(y)=\frac{\|y\|^{2}}{2 \lambda}$ for $\|y\| \leq \lambda$ and $\Phi_{\lambda}(y)=\|y\|-\frac{\lambda}{2}$ for $\|y\|>\lambda$.

(ii) If $\Phi(\cdot)=\|\cdot\|_{1}$, then $\Phi_{\lambda}(y)=\frac{\|y\|_{1}^{2}}{2 \lambda}$ for $\|y\|_{1} \leq \lambda$ and $\Phi_{\lambda}(y)=\|y\|_{1}-\frac{\lambda}{2}$ for $\|y\|_{1}>\lambda$.

Proof.

(i) For each $y \in \mathbb{R}^{n}$, let $R_{y}(z):=\|z\|+\frac{1}{2 \lambda}\|z-y\|^{2}=\|z\|+\frac{1}{2 \lambda}\left(\|z\|^{2}-2\langle z, y\rangle+\right.$ $\left.\|y\|^{2}\right), z \in \mathbb{R}^{n}$. For $\|y\| \leq \lambda$, we have $\left\|\frac{1}{\lambda}\langle z, y\rangle\right\| \leq\|z\|$. Hence, $R_{y}(z) \geq \frac{\|y\|^{2}}{2 \lambda}$ and $\Phi_{\lambda}(y)=R_{y}(0)=\frac{\|y\|^{2}}{2 \lambda}$. For $\|y\|>\lambda$, it is easy to see that $R_{y}(z) \geq$ $\frac{1}{2 \lambda}\left\{\|z\|^{2}-2(\|y\|-\lambda)\|z\|+\|y\|^{2}\right\}=\frac{1}{2 \lambda}(\|z\|-\|y\|+\lambda)^{2}+\|y\|-\frac{\lambda}{2} \geq\|y\|-\frac{\lambda}{2}$. Therefore $\Phi_{\lambda}(y)=R_{y}\left(z^{\prime}\right)=\|y\|-\frac{\lambda}{2}$, where $z^{\prime} \in \mathbb{R}^{n}$ satisfies $\left\langle z^{\prime}, y\right\rangle=\left\|z^{\prime}\right\|\|y\|$ and $\left\|z^{\prime}\right\|+\lambda=\|y\|$.

(ii) Let $R_{y}^{1}(z):=\|z\|_{1}+\frac{1}{2 \lambda}\|z-y\|_{1}^{2}=\left|z_{1}\right|+\cdots+\left|z_{n}\right|+\frac{1}{2 \lambda}\left(\left|z_{1}-y_{1}\right|+\cdots+\right.$ $\left.\left|z_{n}-y_{n}\right|\right)^{2}$. Since we want to minimize $R_{y}^{1}(z)$, we may consider $z \in \mathbb{R}^{n}$, 


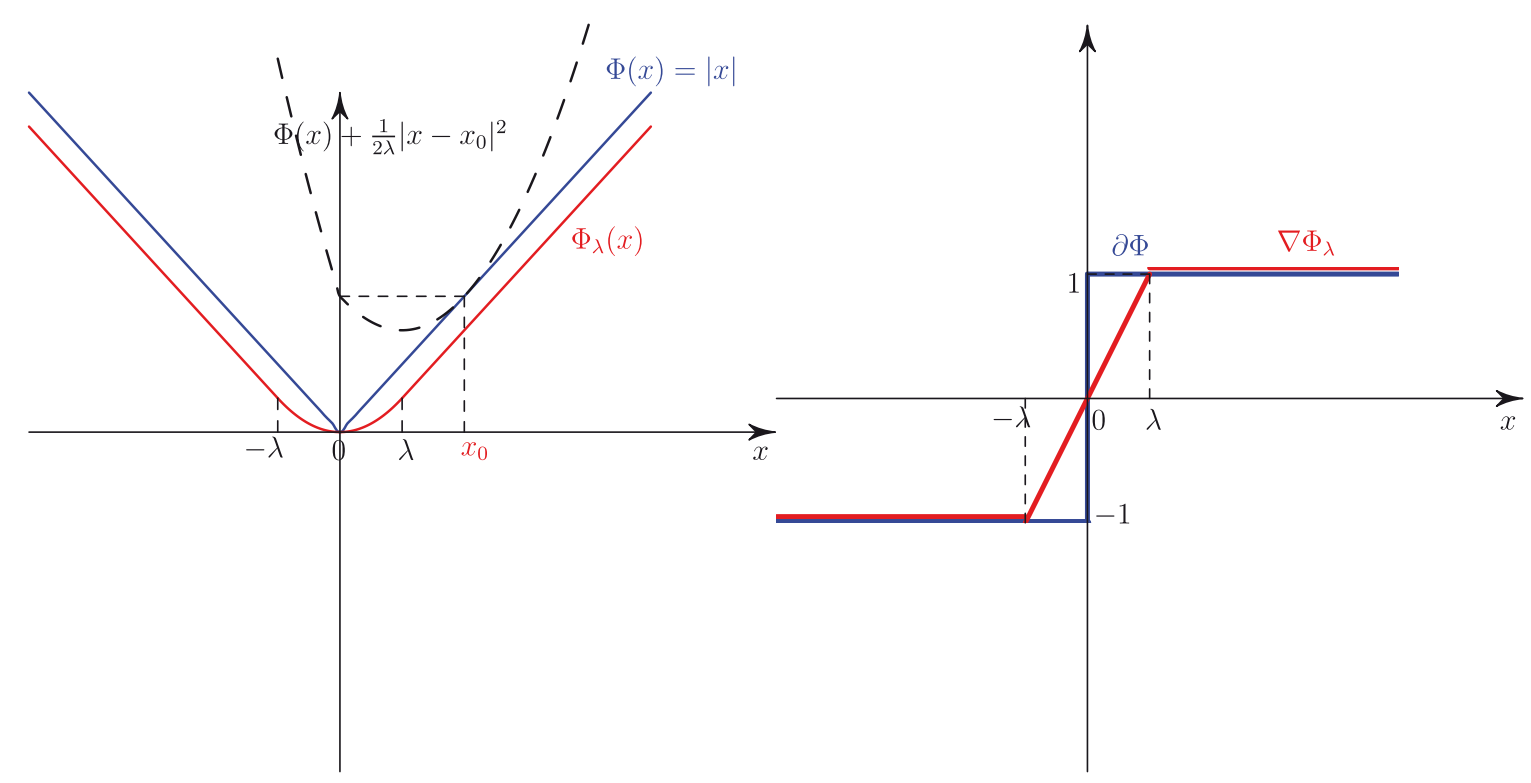

FIG. 3.1. Moreau-Yosida approximation of $\Phi(x)=|x|, x \in \mathbb{R}$.

which satisfies $y_{i} z_{i} \geq 0,\left|z_{i}\right| \leq\left|y_{i}\right|$ for $i=1, \ldots, n\left({ }^{*}\right)$. Then $R_{y}^{1}(z) \geq$ $\left|z_{1}\right|+\cdots+\left|z_{n}\right|+\frac{1}{2 \lambda}\left(\left|y_{1}\right|-\left|z_{1}\right|+\cdots+\left|y_{n}\right|-\left|z_{n}\right|\right)^{2}=\|z\|_{1}+\frac{1}{2 \lambda}\left(\|y\|_{1}-\|z\|_{1}\right)^{2}$. If $\|y\|_{1} \leq \lambda$, then $R_{y}^{1}(z) \geq\|z\|_{1}-\frac{\|y\|_{1}}{\lambda}\|z\|_{1}+\frac{1}{2 \lambda}\left(\|y\|_{1}^{2}+\|z\|_{1}^{2}\right) \geq \frac{\|y\|_{1}^{2}}{2 \lambda}$. Therefore, $\Phi_{\lambda}(y)=R_{y}^{1}(0)=\frac{\|y\|_{1}^{2}}{2 \lambda}$. If $\|y\|_{1}>\lambda$, then $R_{y}^{1}(z) \geq\|z\|_{1}+\frac{1}{2 \lambda}\left(\|y\|_{1}-\|z\|_{1}\right)^{2}=$ $\frac{1}{2 \lambda}\left(\|z\|_{1}-\|y\|_{1}+\lambda\right)^{2}+\|y\|_{1}-\frac{\lambda}{2} \geq\|y\|_{1}-\frac{\lambda}{2}$. Hence, $\Phi_{\lambda}(y)=R_{y}^{1}\left(z^{\prime}\right)=\|y\|_{1}-\frac{\lambda}{2}$, where $z^{\prime}$ satisfies condition $(*)$ and $\left\|z^{\prime}\right\|_{1}+\lambda=\|y\|_{1}$.

Lemma 3.2. Let $\Phi(\cdot)=c\|\cdot\|$ or $\Phi(\cdot)=c\|\cdot\|_{1}$, where $c>0$. Then if $\sup _{\left(t, x_{1}\right) \in \mathbb{R} \times \mathbb{R}^{n}}\left\|F\left(t, x_{1}, \cdot\right)\right\| \leq c \min \left\{1, \frac{\|\cdot\|}{\lambda^{\prime}}\right\}$ for some $\lambda^{\prime}>0$, property $\left(\mathcal{H}_{\Phi, F}\right)$ is satisfied.

Proof. Let us choose $\lambda^{*}:=\lambda^{\prime}$. First, we consider $\Phi(\cdot)=c\|\cdot\|$. Then for $0<\lambda \leq \lambda^{*}, t \geq 0, x \in \mathbb{R}^{n},\|y\|>\lambda$, by using Lemma 3.1, we have $\left\langle\nabla \Phi_{\lambda}(y)+\right.$ $F(t, x, y), y\rangle=\left\langle\frac{c y}{\|y\|}+F(t, x, y), y\right\rangle \geq c\|y\|-c\|y\|=0$. In the case $\|y\| \leq \lambda$, we also have $\left\langle\nabla \Phi_{\lambda}(y)+F(t, x, y), y\right\rangle=\left\langle\frac{c y}{\lambda}+F(t, x, y), y\right\rangle \geq \frac{c\|y\|^{2}}{\lambda^{*}}-\frac{c\|y\|^{2}}{\lambda^{*}}=0$. If $\Phi(\cdot)=c\|\cdot\|_{1}$, the computations can be done similarly. Indeed, for $0<\lambda \leq \lambda^{*}, t \geq 0, x \in \mathbb{R}^{n},\|y\|_{1}>\lambda$, we have $\left\langle\nabla \Phi_{\lambda}(y)+F(t, x, y), y\right\rangle=c\|y\|_{1}+\langle F(t, x, y), y\rangle \geq c\|y\|_{1}-c\|y\| \geq 0$. When $\|y\|_{1} \leq \lambda$, we obtain $\left\langle\nabla \Phi_{\lambda}(y)+F(t, x, y), y\right\rangle=\frac{c\|y\|_{1}^{2}}{\lambda}+\langle F(t, x, y), y\rangle \geq \frac{c\|y\|_{1}^{2}}{\lambda^{*}}-$ $\frac{c\|y\|^{2}}{\lambda^{*}} \geq 0$.

In the scalar case, the Moreau-Yosida approximation is as depicted in Figure 3.1. One recovers the saturation function that is widely used in control applications. Considering the Moreau-Yosida approximation associated with the euclidean norm and 1-norm are interesting because it allows us to study in a systematic way the extension of the saturation function toward codimension $\geq 2$ switching surfaces.

Since $M(q)$ is symmetric positive definite for all $q$, the matrix $M^{-1}(q)$ exists and is also symmetric positive definite for all $q$. Moreover, we have the following lemma.

LeMma 3.3. For all $q \in \mathbb{R}^{n}$,

$$
\frac{1}{k_{2}} \leq\left\|M^{-1}(q)\right\|_{m} \leq \frac{1}{k_{1}},
$$

where the norm here is the induced matrix norm. 
Proof. For all $x \in \mathbb{R}^{n}$, we have

$$
\begin{aligned}
\left\langle M^{-1}(q) x, x\right\rangle & =x^{T} M^{-1}(q) x=x^{T} M^{-1}(q) M(q) M^{-1}(q) x \\
& =\left(M^{-1}(q) x\right)^{T} M(q)\left(M^{-1}(q) x\right) \geq k_{1}\left\|M^{-1}(q) x\right\|^{2} \\
\Rightarrow k_{1}\left\|M^{-1}(q) x\right\|^{2} & \leq\left\langle M^{-1}(q) x, x\right\rangle \leq\left\|M^{-1}(q) x\right\|\|x\| \\
\Rightarrow \frac{\left\|M^{-1}(q) x\right\|}{\|x\|} & \leq \frac{1}{k_{1}} \Rightarrow\left\|M^{-1}(q)\right\|_{m} \leq \frac{1}{k_{1}} .
\end{aligned}
$$

Let $x_{0}$ be an eigenvector of $M^{-1}(q)$ and $\left\|x_{0}\right\|=1$; we have

$$
\left\langle M^{-1}(q) x_{0}, x_{0}\right\rangle=\left\|M^{-1}(q) x_{0}\right\| .
$$

Then

$$
\begin{aligned}
\left\|M^{-1}(q) x_{0}\right\| & =\left\langle M^{-1}(q) x_{0}, x_{0}\right\rangle=\left(M^{-1}(q) x_{0}\right)^{T} M(q)\left(M^{-1}(q) x_{0}\right) \leq k_{2}\left\|M^{-1}(q) x_{0}\right\|^{2} \\
& \Rightarrow\left\|M^{-1}(q) x_{0}\right\| \geq \frac{1}{k_{2}} \Rightarrow\left\|M^{-1}(q)\right\|_{m}=\sup _{\|x\|=1}\left\|M^{-1}(q) x\right\| \geq \frac{1}{k_{2}} .
\end{aligned}
$$

The conclusion follows.

LEMMA 3.4. There exists $k_{5}>0$ such that for all $u, v, w \in \mathbb{R}^{n}$, we have

$$
\left\|M^{-1}(u) w-M^{-1}(v) w\right\| \leq k_{5}\|u-v\|\|w\| .
$$

Proof. We have

$$
\begin{aligned}
& \left\|M^{-1}(u) w-M^{-1}(v) w\right\|=\left\|M^{-1}(u) w-M^{-1}(u) M(u) M^{-1}(v) w\right\| \\
& \quad \leq\left\|M^{-1}(u)\right\|_{m} \cdot\left\|w-M(u) M^{-1}(v) w\right\| \leq \frac{1}{k_{1}}\left\|M(v) M^{-1}(v) w-M(u) M^{-1}(v) w\right\| \\
& \quad \leq \frac{k_{3}}{k_{1}}\|u-v\| \cdot\left\|M^{-1}(v) w\right\| \leq \frac{k_{3}}{k_{1}^{2}}\|u-v\| \cdot\|w\| .
\end{aligned}
$$

So we have the result with $k_{5}=\frac{k_{3}}{k_{1}^{2}}$.

4. Existence of solutions. For any positive real number $\lambda$, we approximate the differential inclusion (1.1) by the following differential equation:

$M\left(q_{\lambda}(t)\right) \ddot{q}_{\lambda}(t)+C\left(q_{\lambda}(t), \dot{q}_{\lambda}(t)\right) \dot{q}_{\lambda}(t)+\nabla \mathcal{V}\left(q_{\lambda}(t)\right)+F\left(t, q_{\lambda}(t), \dot{q}_{\lambda}(t)\right)=-\nabla \Phi_{\lambda}\left(\dot{q}_{\lambda}(t)\right)$,

where $\Phi_{\lambda}(\cdot)$ denotes the Moreau-Yosida approximation (of index $\lambda$ ) of $\Phi(\cdot)$. For all $\lambda>0$, we have $\Phi_{\lambda}(\cdot)$ is $\mathcal{C}^{1}$ and $\nabla \Phi_{\lambda}(\cdot)$ is Lipschitz continuous with constant $\frac{1}{\lambda}$. Without loss of generality, we suppose from now that $t_{0}=0$.

LEMMA 4.1. Let Assumptions 3.1, 3.2, and 3.3 hold and $0<\lambda \leq \lambda^{*}$ ( $\lambda^{*}$ is defined in Assumption 3.3). Then, for every $\left(q_{\lambda 0}, \dot{q}_{\lambda 0}\right) \in \mathbb{R}^{n} \times \mathbb{R}^{n}$, there exists a unique maximal (classical) solution $q_{\lambda}:[0,+\infty) \rightarrow \mathbb{R}^{n}$ satisfying $\left(q_{\lambda}(0), \dot{q}_{\lambda}(0)\right)=\left(q_{0}, \dot{q}_{0}\right)$.

Proof. Let $x_{1}=q_{\lambda}, x_{2}=\dot{q}_{\lambda}$ and $x=\left(x_{1}^{T} x_{2}^{T}\right)^{T}$. We reduce (4.1) to the first order ODE:

$$
\dot{x}=f(t, x),
$$

where $f: \mathbb{R} \times \mathbb{R}^{2 n} \rightarrow \mathbb{R}^{2 n}$ and

$$
f(t, x):=\left(\begin{array}{c}
x_{2} \\
-M^{-1}\left(x_{1}\right)\left[\nabla \Phi_{\lambda}\left(x_{2}\right)+\nabla \mathcal{V}\left(x_{1}\right)+C\left(x_{1}, x_{2}\right) x_{2}+F\left(t, x_{1}, x_{2}\right)\right]
\end{array}\right) .
$$


Let $g: \mathbb{R}^{2 n} \rightarrow \mathbb{R}^{n}$ and

$$
g(x)=g\left(x_{1}, x_{2}\right):=-\left[\nabla \Phi_{\lambda}\left(x_{2}\right)+\nabla \mathcal{V}\left(x_{1}\right)+C\left(x_{1}, x_{2}\right) x_{2}\right]
$$

then $g(\cdot)$ is locally Lipschitz due to the assumptions $\left(\mathcal{H}_{C}\right),\left(\mathcal{H}_{\mathcal{V}}-\mathrm{i}\right)$. Let

$$
G(x)=G\left(x_{1}, x_{2}\right):=M^{-1}\left(x_{1}\right) g(x) .
$$

We will prove that $G(\cdot)$ is also locally Lipschitz. In fact, for all $x, y \in \mathbb{R}^{2 n}$, we have

$$
\begin{aligned}
\|G(x)-G(y)\|= & \left\|M^{-1}\left(x_{1}\right) g(x)-M^{-1}\left(y_{1}\right) g(y)\right\| \leq\left\|M^{-1}\left(x_{1}\right) g(x)-M^{-1}\left(y_{1}\right) g(x)\right\| \\
& +\left\|M^{-1}\left(y_{1}\right) g(x)-M^{-1}\left(y_{1}\right) g(y)\right\| \\
\leq & k_{5}\left\|x_{1}-y_{1}\right\|\|g(x)\|+\left\|M^{-1}\left(y_{1}\right)\right\|_{m}\|g(x)-g(y)\| \\
\leq & k_{5}\left\|x_{1}-y_{1}\right\|\|g(x)\|+\frac{1}{k_{1}}\|g(x)-g(y)\| .
\end{aligned}
$$

Therefore, $G(\cdot)$ is locally Lipschitz. Combining with the assumption $\left(\mathcal{H}_{F}\right)$, we obtain that $f(t, x)$ is continuous in $t$ and locally Lipschitz in $x$. Then for given $\left(q_{\lambda 0}, \dot{q}_{\lambda 0}\right)$, there exists uniquely a $\mathcal{C}^{1}$ local solution for (4.1), or equivalently (4.2) (Cauchy-Lipschitz theorem). Let $\left(q_{\lambda}(t), \dot{q}_{\lambda}(t)\right)$ be the maximal solution defined on some interval $\left[0, T_{\max }\right)$ with $0<T_{\max } \leq+\infty$. Consider the energy function, which is the sum of the kinetic energy and the (virtual) potential energy:

$$
V\left(q_{\lambda}, \dot{q}_{\lambda}\right):=\frac{1}{2}\left\langle M\left(q_{\lambda}\right) \dot{q}_{\lambda}, \dot{q}_{\lambda}\right\rangle+\mathcal{V}\left(q_{\lambda}\right)
$$

Then the derivative of $V(\cdot)$ along the trajectories of $(4.1)$ is

$$
\begin{aligned}
\dot{V}\left(q_{\lambda}(t), \dot{q}_{\lambda}(t)\right)= & \frac{1}{2}\left\langle\frac{d}{d t}\left(M\left(q_{\lambda}(t)\right)\right) \dot{q}_{\lambda}(t), \dot{q}_{\lambda}(t)\right\rangle+\left\langle M\left(q_{\lambda}(t)\right) \dot{q}_{\lambda}(t), \ddot{q}_{\lambda}(t)\right\rangle \\
& +\left\langle\nabla \mathcal{V}\left(q_{\lambda}(t)\right), \dot{q}_{\lambda}(t)\right\rangle \\
= & \frac{1}{2}\left\langle\frac{d}{d t}\left(M\left(q_{\lambda}(t)\right)\right) \dot{q}_{\lambda}(t), \dot{q}_{\lambda}(t)\right\rangle-\left\langle C\left(q_{\lambda}, \dot{q}_{\lambda}\right) \dot{q}_{\lambda}+\nabla \mathcal{V}\left(q_{\lambda}\right)+\nabla \Phi_{\lambda}\left(\dot{q}_{\lambda}\right)\right. \\
& \left.+F\left(t, q_{\lambda}(t), \dot{q}_{\lambda}(t)\right), \dot{q}_{\lambda}(t)\right\rangle+\left\langle\nabla \mathcal{V}\left(q_{\lambda}(t)\right), \dot{q}_{\lambda}(t)\right\rangle \\
= & \frac{1}{2}\left\langle\left[\frac{d}{d t}\left(M\left(q_{\lambda}(t)\right)\right)-2 C\left(q_{\lambda}, \dot{q}_{\lambda}\right)\right] \dot{q}_{\lambda}(t), \dot{q}_{\lambda}(t)\right\rangle-\left\langle\nabla \Phi_{\lambda}\left(\dot{q}_{\lambda}(t)\right)\right. \\
& \left.+F\left(t, q_{\lambda}(t), \dot{q}_{\lambda}(t)\right), \dot{q}_{\lambda}(t)\right\rangle \\
= & -\left\langle\nabla \Phi_{\lambda}\left(\dot{q}_{\lambda}(t)\right)+F\left(t, q_{\lambda}(t), \dot{q}_{\lambda}(t)\right), \dot{q}_{\lambda}(t)\right\rangle \leq 0
\end{aligned}
$$

for almost all $t \geq 0$, where Assumption $3.2\left(\mathcal{C}_{2}\right)$ and Assumption $3.3\left(\mathcal{H}_{\Phi, F}\right)$ are used. Therefore for all $t \geq 0$, from Assumption $3.1\left(\mathcal{M}_{1}\right)$ and Assumption $3.3\left(\mathcal{H}_{\mathcal{V}}-\right.$ ii $)$, we obtain

$$
\begin{aligned}
\frac{1}{2} k_{1}\left\|\dot{q}_{\lambda}(t)\right\|^{2} & \leq \frac{1}{2}\left\langle M\left(q_{\lambda}(t)\right) \dot{q}_{\lambda}(t), \dot{q}_{\lambda}(t)\right\rangle=V\left(q_{\lambda}(t), \dot{q}_{\lambda}(t)\right)-\mathcal{V}\left(q_{\lambda}\right)(t) \\
& \leq V\left(q_{0}, \dot{q}_{0}\right)-\inf \mathcal{V}
\end{aligned}
$$

which implies that $\dot{q}_{\lambda}$ is bounded. Assuming that $T_{\max }<+\infty$, we have

$$
\left\|q_{\lambda}(t)\right\| \leq\left\|q_{0}\right\|+T_{\max } \sup _{t \in\left[0, T_{\max }\right)}\left\|\dot{q}_{\lambda}\right\|<+\infty
$$

Hence, $\left(q_{\lambda}(t), \dot{q}_{\lambda}(t)\right)$ is bounded on $\left[0, T_{\max }\right)$. Our result follows by contradiction. 
Theorem 4.2. Let Assumptions 3.1, 3.2, and 3.3 hold. Then, for every $\left(q_{0}, \dot{q}_{0}\right) \in$ $\mathbb{R}^{n} \times \mathbb{R}^{n}$, there exists a solution $q:[0,+\infty) \rightarrow \mathbb{R}^{n}$ of $(1.1)$ in the following sense:

(a) $q \in \mathcal{C}^{1}\left([0,+\infty), \mathbb{R}^{n}\right) \cap \mathcal{W}^{2, \infty}\left([0, T], \mathbb{R}^{n}\right)$ for every $T>0$.

(b) (1.1) is satisfied for a.e. $t \in[0,+\infty)$.

(c) $q(0)=q_{0}$ and $\dot{q}(0)=\dot{q}_{0}$.

Proof. Consider $\lambda \in\left[0, \lambda^{*}\right]$. From (4.7), we have

$\left(\dot{q}_{\lambda}\right)$ is uniformly bounded in $\mathrm{L}^{\infty}\left([0,+\infty), \mathbb{R}^{\mathrm{n}}\right)$.

Fix $T>0$. From $q_{\lambda}(t)=q_{0}+\int_{0}^{t} \dot{q}_{\lambda}(s) d s$ we obtain

$\left(q_{\lambda}\right)$ is uniformly bounded in $\mathrm{L}^{\infty}\left([0, \mathrm{~T}], \mathbb{R}^{\mathrm{n}}\right)$.

Because $\nabla \mathcal{V}(\cdot)$ is bounded on bounded sets, we deduce

$\left(\nabla \mathcal{V}\left(q_{\lambda}\right)\right)$ is uniformly bounded in $\mathrm{L}^{\infty}\left([0, \mathrm{~T}], \mathbb{R}^{\mathrm{n}}\right)$

Furthermore, from Assumption $3.2\left(\mathcal{C}_{1}\right)$, we have $\left\|C\left(q_{\lambda}(t), \dot{q}_{\lambda}(t)\right) \dot{q}_{\lambda}(t)\right\| \leq k_{4}\left\|\dot{q}_{\lambda}(t)\right\|^{2}$, which implies

$$
\left(C\left(q_{\lambda}(t), \dot{q}_{\lambda}\right) \dot{q}_{\lambda}\right) \text { is uniformly bounded in } \mathrm{L}^{\infty}\left([0, \mathrm{~T}], \mathbb{R}^{\mathrm{n}}\right)
$$

Finally, it is classical that for all $x \in \mathbb{R}^{n},\left\|\nabla \Phi_{\lambda}(x)\right\| \leq\|m(\partial \Phi(x))\|$, where $m(\partial \Phi(x))$ is the element of minimal norm. Hence, $\left\|\nabla \Phi_{\lambda}\left(\dot{q}_{\lambda}\right)\right\| \leq\left\|m\left(\partial \Phi\left(\dot{q}_{\lambda}\right)\right)\right\|$. Note that since $\partial \Phi(\cdot)$ is bounded on bounded sets, we obtain

$$
\left(\nabla \Phi_{\lambda}\left(\dot{q}_{\lambda}\right)\right) \text { is uniformly bounded in } \mathrm{L}^{\infty}\left([0, \mathrm{~T}], \mathbb{R}^{\mathrm{n}}\right)
$$

From (1.1) and Assumption $3.3\left(\mathcal{H}_{F}\right)$, we have

$$
\left(\ddot{q}_{\lambda}\right) \text { is uniformly bounded in } \mathrm{L}^{\infty}\left([0, \mathrm{~T}], \mathbb{R}^{\mathrm{n}}\right) \text {. }
$$

Then there exists a function $q \in \mathcal{C}^{1}\left([0, T], \mathbb{R}^{n}\right) \cap \mathcal{W}^{2,+\infty}\left([0, T], \mathbb{R}^{n}\right)$ and a subsequence of $\left(q_{\lambda}\right)$, still denoted by $\left(q_{\lambda}\right)$ such that $q_{\lambda}(\cdot), \dot{q}_{\lambda}(\cdot)$ converge uniformly to $q(\cdot), \dot{q}(\cdot)$, respectively, and $\ddot{q}_{\lambda}(\cdot)$ converges to $\ddot{q}(\cdot)$ for the topology $\sigma\left(L^{\infty}\left([0, T], \mathbb{R}^{n}\right), L^{1}\left([0, T], \mathbb{R}^{n}\right)\right)$ (see [1]). We prove that $q(\cdot)$ satisfies $(1.1)$ for a.e. $t$ on $[0, T]$. With the same arguments as in [1], it is sufficient to prove that

$M\left(q_{\lambda}\right) \ddot{q}_{\lambda}+C\left(q_{\lambda}, \dot{q}_{\lambda}\right) \dot{q}_{\lambda}+\nabla \mathcal{V}\left(q_{\lambda}\right)+F\left(\cdot, q_{\lambda}, \dot{q}_{\lambda}\right) \rightarrow M(q) \ddot{q}+C(q, \dot{q}) \dot{q}+\nabla \mathcal{V}(q)+F(\cdot, q, \dot{q})$

for the topology $\sigma\left(L^{\infty}, L^{1}\right)$.

Since $q_{\lambda} \rightarrow q$ uniformly and $\nabla \mathcal{V}(\cdot)$ is Lipschitz continuous on the bounded sets, we have $\nabla \mathcal{V}\left(q_{\lambda}\right) \rightarrow \nabla \mathcal{V}(q)$ uniformly in $\mathcal{C}\left([0, T], \mathbb{R}^{n}\right)$. From Assumption $3.3\left(\mathcal{H}_{C}\right),\left(\mathcal{H}_{F}\right)$ and the fact that $q_{\lambda} \rightarrow q, \dot{q}_{\lambda} \rightarrow \dot{q}$, we obtain that $C\left(q_{\lambda}, \dot{q}_{\lambda}\right) \dot{q}_{\lambda}+F\left(\cdot, q_{\lambda}, \dot{q}_{\lambda}\right)$ converges to $C(q, \dot{q}) \dot{q}+F(\cdot, q, \dot{q})$ uniformly. Finally, we will check that $M\left(q_{\lambda}\right) \ddot{q}_{\lambda}$ weakly converges to $M(q) \ddot{q}$ in the $\sigma\left(L^{\infty}, L^{1}\right)$ sense. Note that from Assumption $3.1\left(\mathcal{M}_{1}\right)$

$$
\|M(q(t)) \ddot{q}(t)\| \leq\|M(q(t))\|_{m}\|\ddot{q}(t)\| \leq k_{2}\|\ddot{q}(t)\|,
$$

which means $M(q) \ddot{q} \in L^{\infty}\left([0, T], \mathbb{R}^{n}\right)$. Similarly, we have $M\left(q_{\lambda}\right) \ddot{q}_{\lambda} \in L^{\infty}\left([0, T], \mathbb{R}^{n}\right)$. For every $\varphi \in L^{1}\left([0, T], \mathbb{R}^{n}\right)$ we have $M(q) \varphi \in L^{1}\left([0, T], \mathbb{R}^{n}\right)$ and 


$$
\begin{aligned}
& \left|\int_{0}^{T}\left\langle M\left(q_{\lambda}(t)\right) \ddot{q}_{\lambda}(t)-M(q)(t) \ddot{q}(t), \varphi(t)\right\rangle d t\right| \\
& \quad \leq\left|\int_{0}^{T}\left\langle\left[M\left(q_{\lambda}(t)\right)-M(q(t))\right] \ddot{q}_{\lambda}(t), \varphi(t)\right\rangle d\right|+\left|\int_{0}^{T}\left\langle M(q(t))\left(\ddot{q}_{\lambda}(t)-\ddot{q}(t)\right), \varphi(t)\right\rangle d t\right| \\
& \quad \leq k_{3} \int_{0}^{T}\left\|q_{\lambda}(t)-q(t)\right\|\left\|\ddot{q}_{\lambda}(t)\right\|\|\varphi(t)\| d t+\left|\int_{0}^{T}\left\langle\left(\ddot{q}_{\lambda}(t)-\ddot{q}(t)\right), M(q(t)) \varphi(t)\right\rangle d t\right| \rightarrow 0
\end{aligned}
$$

when $\lambda \rightarrow 0$ since $\ddot{q}_{\lambda}$ converges weakly to $\ddot{q}$ and $q_{\lambda}$ converges to $q$ uniformly. Hence, $M\left(q_{\lambda}\right) \ddot{q}_{\lambda}$ converges weakly to $M(q) \ddot{q}$. Hence, we obtain $M\left(q_{\lambda}\right) \ddot{q}_{\lambda}+C\left(q_{\lambda}, \dot{q}_{\lambda}\right) \dot{q}_{\lambda}+$ $\nabla \mathcal{V}\left(q_{\lambda}\right)+F\left(\cdot, q_{\lambda}, \dot{q}_{\lambda}\right)$ converges weakly to $M(q) \ddot{q}+C(q, \dot{q}) \dot{q}+\nabla \mathcal{V}(q)+F(\cdot, q, \dot{q})$ in the $\sigma\left(L^{\infty}, L^{1}\right)$ sense. Since $T>0$ can be chosen arbitrarily, we have proved the theorem.

5. Uniqueness of solutions. Let us start with a result which relies on the assumption that the matrix $M^{-1}(q)$ does not destroy the monotonicity of the operator $\partial \Phi(\cdot)$.

TheOrem 5.1. Let Assumptions 3.1, 3.2, and 3.3 hold. Moreover, assume that for all $m, p \in \mathbb{R}^{n}$, there exist $\varepsilon>0, \gamma>0$ such that for all $p_{1}, p_{2} \in \mathbb{B}_{\varepsilon}(p)$, we have

$$
\left\langle M^{-1}(m)\left(p_{1}^{*}-p_{2}^{*}\right), p_{1}-p_{2}\right\rangle \geq-\gamma\left\|p_{1}-p_{2}\right\|^{2} \forall p_{1}^{*} \in \partial \Phi\left(p_{1}\right) \forall p_{2}^{*} \in \partial \Phi\left(p_{2}\right) .
$$

Then (1.1) has a unique solution in the sense of Theorem 4.2.

Proof. For arbitrary $T>0$, suppose that $\left(q_{1}(\cdot), \dot{q}_{1}(\cdot)\right),\left(q_{2}(\cdot), \dot{q}_{1}(\cdot)\right)$ are two solutions of $(1.1)$ on $[0, T]$ with the same initial conditions. Let $z_{i}:=\left(\begin{array}{l}q_{i}^{T} \dot{q}_{i}^{T}\end{array}\right)^{T}$ for $i=1,2$; then $z_{1}(\cdot)$ and $z_{2}(\cdot)$ are two solutions of the differential inclusion,

$$
\dot{x}(t)+A(t, x(t)) \in-B(x(t)),
$$

where $x=\left(x_{1}^{T} x_{2}^{T}\right)^{T}, B(x):=\left(\begin{array}{c}0 \\ M^{-1}\left(x_{1}\right) \partial \Phi\left(x_{2}\right)\end{array}\right)$ and

$$
A(t, x):=\left(\begin{array}{c}
-x_{2} \\
M^{-1}\left(x_{1}\right)\left[\nabla \mathcal{V}\left(x_{1}\right)+C\left(x_{1}, x_{2}\right) x_{2}+F\left(t, x_{1}, x_{2}\right)\right]
\end{array}\right) .
$$

As we know, $A(t, x)$ is continuous in $t$ and uniformly locally Lipschitz in $x$. We now prove that $B(\cdot)$ is locally hypomonotone. Indeed, for all $m, p \in \mathbb{R}^{2 n}, m=$ $\left(m_{1}^{T} m_{2}^{T}\right)^{T}, p=\left(p_{1}^{T} p_{2}^{T}\right)^{T}, m_{2}^{*} \in \partial \Phi\left(m_{2}\right), p_{2}^{*} \in \partial \Phi\left(p_{2}\right)$ we have

$$
\begin{aligned}
& \left\langle M^{-1}\left(m_{1}\right) m_{2}^{*}-M^{-1}\left(p_{1}\right) p_{2}^{*}, m_{2}-p_{2}\right\rangle \\
& \quad=\left\langle M^{-1}\left(m_{1}\right)\left(m_{2}^{*}-p_{2}^{*}\right), m_{2}-p_{2}\right\rangle+\left\langle\left(M^{-1}\left(m_{1}\right)-M^{-1}\left(p_{1}\right)\right) p_{2}^{*}, m_{2}-p_{2}\right\rangle \\
& \quad \geq-\gamma\left\|m_{2}-p_{2}\right\|^{2}-k_{5}\left\|p_{2}^{*}\right\|\left\|m_{1}-p_{1}\right\|\left\|m_{2}-p_{2}\right\|,
\end{aligned}
$$

where (5.1) has been used to obtain the first inequality. Therefore $B(\cdot)$ is locally hypomonotone due to the boundedness of $\partial \Phi(\cdot)$ on bounded sets. Then, there exists a $\sigma>0$ such that $A(t, \cdot)$ is uniformly Lipschitz with constant $l_{1}>0$ and $B(\cdot)$ is hypomonotone with constant $l_{2}>0$ in $\mathbb{B}_{\sigma}\left(z_{0}\right)$, where $z_{0}=\left(q_{0}^{T} \dot{q}_{0}^{T}\right)^{T}$. Due to the continuity of $z_{1}(\cdot), z_{2}(\cdot)$, there exists a $T_{0}$ such that $0<T_{0}<T$ and $z_{1}(t), z_{2}(t) \in$ $\mathbb{B}_{\sigma}\left(z_{0}\right)$ for all $t \in\left[0, T_{0}\right]$. For almost all $t \in\left[0, T_{0}\right]$, we have

$$
\left\langle\dot{z}_{1}(t)-\dot{z}_{2}(t)+A\left(t, z_{1}(t)\right)-A\left(t, z_{2}(t)\right), z_{1}(t)-z_{2}(t)\right\rangle \leq l_{2}\left\|z_{1}(t)-z_{2}(t)\right\|^{2},
$$


which implies

$$
\begin{aligned}
\frac{1}{2} \frac{d}{d t}\left\|z_{1}(t)-z_{2}(t)\right\|^{2} & \leq\left\langle A\left(t, z_{2}(t)\right)-A\left(t, z_{1}(t)\right), z_{1}(t)-z_{2}(t)\right\rangle+l_{2}\left\|z_{1}(t)-z_{2}(t)\right\|^{2} \\
& \leq\left(l_{1}+l_{2}\right)\left\|z_{1}(t)-z_{2}(t)\right\|^{2}=l\left\|z_{1}(t)-z_{2}(t)\right\|^{2}
\end{aligned}
$$

where $l=l_{1}+l_{2}$. By Gronwall's inequality, we have $\left\|z_{1}(t)-z_{2}(t)\right\|^{2} \leq 0$ for all $t \in\left[0, T_{0}\right]$ or $z_{1}(t)=z_{2}(t)$ for all $t \in\left[0, T_{0}\right]$. Now, we suppose there exists $t_{1} \in[0, T)$ such that $z_{1}\left(t_{1}\right) \neq z_{2}\left(t_{1}\right)$. Let

$$
E:=\left\{t \in\left[0, t_{1}\right]: z_{1}(t) \neq z_{2}(t)\right\}
$$

Since $t_{1} \in E$ and $E$ is bounded from below, there exists $\alpha=\inf E$, where $\alpha \in\left(t_{0}, t_{1}\right]$ and for all $t \in\left[t_{0}, \alpha\right): z_{1}(t)=z_{2}(t)$. By the continuity of $z_{1}(\cdot)$ and $z_{2}(\cdot)$, we have $z_{1}(\alpha)=z_{2}(\alpha)$, which implies that $\alpha<t_{1}$. With the same argument as above, there exists a neighborhood of $\alpha$ such that $z_{1}(\cdot) \equiv z_{2}(\cdot)$. This is a contradiction. So $z_{1}(\cdot) \equiv z_{2}(\cdot)$ on $[0, T]$ and hence, $q_{1}(\cdot) \equiv q_{2}(\cdot)$ on $[0, T]$.

The following propositions give some cases where $M(q)$ and $\Phi(\cdot)$ are such that (5.1) is satisfied.

Proposition 5.2. Suppose that for each $q \in \mathbb{R}^{n}, M(q)$ is a positive definite diagonal matrix and $\Phi(q)=\Phi_{1}\left(q_{1}\right)+\cdots+\Phi_{n}\left(q_{n}\right)$, where $\Phi_{i}: \mathbb{R} \rightarrow \mathbb{R}$ is a convex function, $i=1, \ldots, n$. Then (5.1) holds.

Proof. Let $x, y, z \in \mathbb{R}^{n}$ and $y^{*} \in \partial \Phi(y), z^{*} \in \partial \Phi(z)$. Suppose that $M^{-1}(x)=$ $\operatorname{diag}\left(k_{1}(x), \ldots, k_{n}(x)\right)$, where $k_{i}(x)>0, i=1,2, \ldots, n$. Then, we obtain

$$
\begin{aligned}
& \left\langle M^{-1}(x)\left(y^{*}-z^{*}\right), y-z\right\rangle \\
& =k_{1}(x)\left(y_{1}^{*}-z_{1}^{*}\right) \cdot\left(y_{1}-z_{1}\right)+\cdots+k_{n}(x)\left(y_{n}^{*}-z_{n}^{*}\right) \cdot\left(y_{n}-z_{n}\right) \geq 0
\end{aligned}
$$

where $y_{i}^{*} \in \partial \Phi_{i}\left(y_{i}\right), z_{i}^{*} \in \partial \Phi_{i}\left(z_{i}\right), i=1,2, \ldots, n$.

Proposition 5.3. Suppose that for each $q=\left(q_{1} q_{2} \ldots q_{n}\right)^{T} \in \mathbb{R}^{n}$,

(1) $M(q)=\left(\begin{array}{cc}M_{1}(q) & 0 \\ 0 & M_{2}(q)\end{array}\right)$, where $M_{1}(q) \in \mathbb{R}^{m \times m}$, and $M_{2}(q) \in \mathbb{R}^{p \times p}$ is a positive definite diagonal matrix, $n=m+p$.

(2) $\Phi(q)=\Phi_{m+1}\left(q_{m+1}\right)+\cdots+\Phi_{n}\left(q_{n}\right)$, where $\Phi_{i}: \mathbb{R} \rightarrow \mathbb{R}$ is a convex function, $i=m+1, \ldots, n$. Then the condition (5.1) is satisfied.

Proof. Let $x, y, z \in \mathbb{R}^{n}$ and $y^{*} \in \partial \Phi(y), z^{*} \in \partial \Phi(z)$. Assume that $M_{2}^{-1}(x)=$ $\operatorname{diag}\left(k_{m+1}(x), \ldots, k_{n}(x)\right)$, where $k_{i}(x)>0, i=m+1, \ldots, n$. Then, we have

$$
\begin{aligned}
& \left\langle M^{-1}(x)\left(y^{*}-z^{*}\right), y-z\right\rangle \\
& \quad=k_{m+1}(x)\left(y_{m+1}^{*}-z_{m+1}^{*}\right) \cdot\left(y_{m+1}-z_{m+1}\right)+\cdots+k_{n}(x)\left(y_{n}^{*}-z_{n}^{*}\right) \cdot\left(y_{n}-z_{n}\right) \geq 0,
\end{aligned}
$$

where $y_{i}^{*} \in \partial \Phi_{i}\left(y_{i}\right), z_{i}^{*} \in \partial \Phi_{i}\left(z_{i}\right), i=m+1, \ldots, n$.

Remark 5.1.

(i) We also have the uniqueness result if we replace assumption (2) in Proposition 5.3 by the following:

$\left(2^{\prime}\right) \Phi(q)=\Psi\left(q_{1}, \ldots, q_{m}\right)+\Phi_{m+1}\left(q_{m+1}\right)+\cdots+\Phi_{n}\left(q_{n}\right)$, where $\Phi_{i}: \mathbb{R} \rightarrow \mathbb{R}$ is a convex function, $i=m+1, \ldots, n, \Psi \in \mathcal{C}^{1}\left(\mathbb{R}^{m} ; \mathbb{R}\right)$ is convex, and $\nabla \Psi$ is locally Lipschitz.

Indeed, we can put the term $\nabla \Psi(\cdot)$ into $A(\cdot)$ and the new function $A(\cdot)$ is still locally Lipschitz (in $x$ ).

(ii) If $M$ is a constant matrix, then the change of coordinates that has been used in $[2,14,15,16]$ can be used to prove the uniqueness by setting $z=M^{\frac{1}{2}} q$ and $\varphi(\cdot)=\Phi \circ M^{-\frac{1}{2}}(\cdot)$. 
Let us note that other results for the uniqueness of solutions exist, like Filippov's criterion for codimension one attractive surfaces [20]. Filippov's criterion may apply to (1.1) for specific choices of $\Phi(\cdot)$, for example, $\Phi(\cdot)=\left|D^{T} \circ \cdot\right|$, as in the following proposition. This result shows the link between our development and the sliding mode in control.

Proposition 5.4. If for all $y \in \mathbb{R}^{n}, \Phi(y)=\left|D^{T} y\right|$, where $D \neq 0$ is a vector in $\mathbb{R}^{n}$, then for any initial condition, the solution of (1.1) in the sense of Theorem 4.2 is unique.

Proof. It is easy to compute that $\partial \Phi(y)=D \operatorname{Sign}\left(D^{T} y\right)$. Let $h: \mathbb{R}^{2 n} \rightarrow \mathbb{R}, h(x)=$ $D^{T} x_{2}$, where $x=\left(x_{1}^{T} x_{2}^{T}\right)^{T}$ and

$\Sigma:=\left\{x \in \mathbb{R}^{2 n}: h(x)=0\right\}, S^{-}:=\left\{x \in \mathbb{R}^{2 n}: h(x)<0\right\}, S^{+}:=\left\{x \in \mathbb{R}^{2 n}: h(x)>0\right\}$.

Then (1.1) is equivalent to the first order system

$$
\dot{x} \in \Gamma(x, t):=\left\{\begin{array}{l}
\Gamma^{-}(x, t) \text { if } x \in S^{-}, \\
\overline{\operatorname{co}}\left\{\Gamma^{-}(x, t), \Gamma^{+}(x, t)\right\} \text { if } x \in \Sigma, \\
\Gamma^{+}(x, t) \text { if } x \in S^{+},
\end{array}\right.
$$

where $\Gamma^{-}, \Gamma^{+}: \mathbb{R}^{2 n+1} \rightarrow \mathbb{R}$ are defined by

$$
\Gamma^{-}(x, t):=\left(\begin{array}{c}
x_{2} \\
-M^{-1}\left(x_{1}\right)\left[\nabla \mathcal{V}\left(x_{1}\right)+C\left(x_{1}, x_{2}\right) x_{2}+F\left(t, x_{1}, x_{2}\right)-D\right]
\end{array}\right)
$$

and

$$
\Gamma^{+}(x, t):=\left(\begin{array}{c}
x_{2} \\
-M^{-1}\left(x_{1}\right)\left[\nabla \mathcal{V}\left(x_{1}\right)+C\left(x_{1}, x_{2}\right) x_{2}+F\left(t, x_{1}, x_{2}\right)+D\right]
\end{array}\right) .
$$

Note that for such a system, our solutions (in the sense of Theorem 4.2) and Filippov's solutions coincide. Indeed, if $(y(\cdot) \dot{y}(\cdot))$ is a solution of (5.4) in the sense of Theorem 4.2, then $(y(\cdot) \dot{y}(\cdot))$ is absolutely continuous and satisfies (5.4) for a.e. $t \geq 0$. Therefore, it is also a Filippov solution. Inversely, let $(y(\cdot) \dot{y}(\cdot))$ be a Filippov solution of (5.4). Then $y \in \mathcal{C}^{1}\left([0,+\infty), \mathbb{R}^{n}\right)$ and $(y(\cdot) \dot{y}(\cdot))$ is bounded on $[0, T]$ for all $T>0$. From $(5.4)$, we have $\ddot{y}(\cdot)$ is bounded a.e. on $[0, T]$. Hence $(y(\cdot) \dot{y}(\cdot))$ is a solution of (5.4) in the sense of Theorem 4.2.

$$
\begin{aligned}
\text { Let } n & :=\nabla h=\left(\begin{array}{l}
0 \\
D
\end{array}\right) \text { and } \\
\Gamma_{n}^{-}(x, t) & :=\left\langle\Gamma^{-}(x, t), n\right\rangle \\
& =\left\langle-M^{-1}\left(x_{1}\right)\left[\nabla \mathcal{V}\left(x_{1}\right)+C\left(x_{1}, x_{2}\right) x_{2}+F\left(t, x_{1}, x_{2}\right)\right], D\right\rangle+\left\langle M^{-1}\left(x_{1}\right) D, D\right\rangle, \\
\Gamma_{n}^{+}(x, t) & :=\left\langle\Gamma^{-}(x, t), n\right\rangle \\
& =\left\langle-M^{-1}\left(x_{1}\right)\left[\nabla \mathcal{V}\left(x_{1}\right)+C\left(x_{1}, x_{2}\right) x_{2}+F\left(t, x_{1}, x_{2}\right)\right], D\right\rangle-\left\langle M^{-1}\left(x_{1}\right) D, D\right\rangle .
\end{aligned}
$$

Then

$$
\Gamma_{n}^{-}(x, t)-\Gamma_{n}^{+}(x, t)=2\left\langle M^{-1}\left(x_{1}\right) D, D\right\rangle>0 .
$$

From Theorem 2 in [20, p. 110], uniqueness of solutions follows.

Remark 5.2. Let us note that in general for switching surfaces of codimension $\geq 2$, Filippov's differential inclusions do not have unique solutions, even if the switching surface is attractive. Example of nonuniqueness with codimension 2 attractive surface can be found in [27]. Our study of uniqueness makes sense if one is interested in this property. 
6. Stability analysis. In this section, the Lyapunov stability of equilibria and attractivity of the equilibrium set of (1.1) are investigated. It is remarkable that from here, we do not need the uniqueness of solutions. We make the following assumption through this section and the next one.

Assumption 6.1.

$$
F\left(t, x_{1}, x_{2}\right) \equiv F\left(x_{1}, x_{2}\right),\left(t, x_{1}, x_{2}\right) \in \mathbb{R} \times \mathbb{R}^{n} \times \mathbb{R}^{n}
$$

The equilibrium set $\mathcal{W}$ of $(1.1)$ is given by

$$
\mathcal{W}:=\left\{s \in \mathbb{R}^{n}: \nabla \mathcal{V}(s)+F(s, 0) \in-\partial \Phi(0)\right\}
$$

Assumption 6.2.

$$
\nabla \mathcal{V}(0)+F(0,0) \in-\partial \Phi(0)
$$

Then, we have $0 \in \mathcal{W}$. We reduce (1.1) into the first order differential inclusion,

$$
\dot{x}(t) \in \mathcal{F}(x(t)),
$$

where $x=\left(x_{1}^{T} x_{2}^{T}\right)^{T}$ and

$$
\mathcal{F}(x):=\left(\begin{array}{c}
x_{2} \\
-M^{-1}\left(x_{1}\right)\left[\nabla \mathcal{V}\left(x_{1}\right)+C\left(x_{1}, x_{2}\right) x_{2}+\partial \Phi\left(x_{2}\right)+F\left(x_{1}, x_{2}\right)\right]
\end{array}\right)
$$

It is easy to check that

$$
\mathcal{Y}=\mathcal{W} \times\{0\}
$$

where $\mathcal{Y}$ is the set of stationary solutions of (6.4). Let us recall some definitions about the stability of an equilibrium point in the sense of Lyapunov. For $x_{0} \in \mathbb{R}^{n}$, denote $x\left(t ; x_{0}\right)$ by a solution of $(6.4)$ satisfying the initial condition $x(0)=x_{0}$.

DEFINITION 6.1. The equilibrium point $x=0$ is said to be stable if

$$
\forall \varepsilon>0, \exists \delta(\varepsilon)>0 \text { such that } \forall x_{0} \in \mathbb{R}^{2 n} \text { and }\left\|x_{0}\right\| \leq \delta(\varepsilon) \Rightarrow\left\|x\left(t ; x_{0}\right)\right\| \leq \varepsilon \forall t \geq 0 \text {. }
$$

DEFINITION 6.2. The equilibrium point $x=0$ is said to be attractive if

$$
\exists \delta>0 \text { such that } \forall x_{0} \in \mathbb{R}^{2 n} \text { and }\left\|x_{0}\right\| \leq \delta \Rightarrow \lim _{t \rightarrow \infty}\left\|x\left(t ; x_{0}\right)\right\|=0 .
$$

If this is true for all $x_{0} \in \mathbb{R}^{2 n}$, then $x=0$ is said to be globally attractive.

The next result is an extension of the Lagrange-Dirichlet theorem of mechanics (also called the Lejeune-Dirichlet theorem), where $\mathcal{V}(q)$ is the potential energy, while $\partial \Phi(\dot{q})$ is the dissipation term.

Assumption 6.3.

(a) There exists an $\alpha \geq 0$ such that

$$
\Phi(\cdot) \geq \alpha\|\cdot\| \quad \text { and } \quad \sup _{\left(x_{1}, x_{2}\right) \in \times \mathbb{R}^{n} \times \mathbb{R}^{n}}\left\|F\left(x_{1}, x_{2}\right)\right\| \leq \alpha .
$$

(b) There exist $\alpha>\beta \geq 0$ such that

$$
\Phi(\cdot) \geq \alpha\|\cdot\| \quad \text { and } \quad \sup _{\left(x_{1}, x_{2}\right) \in \times \mathbb{R}^{n} \times \mathbb{R}^{n}}\left\|F\left(x_{1}, x_{2}\right)\right\| \leq \beta .
$$


Remark 6.1. If $F \equiv 0$, then $\alpha=0$ and Assumption 6.3(a) holds. Note that the condition $0 \in \operatorname{int}(\partial \Phi(0))$ is equivalent to $\Phi(\cdot) \geq \alpha\|\cdot\|$ for some $\alpha>0$ (see [1]).

TheOREM 6.3 (stability). Let the assumptions of Theorem 4.2 and Assumptions 6.1, 6.2, and 6.3 hold. Suppose that $\mathcal{V}(\cdot)$ is locally positive definite. Then the origin of the system (6.4) is stable.

Proof. Consider the Lyapunov function $V: \mathbb{R}^{2 n} \rightarrow \mathbb{R}$ defined by

$$
V(x):=\frac{1}{2}\left\langle M\left(x_{1}\right) x_{2}, x_{2}\right\rangle+\mathcal{V}\left(x_{1}\right) .
$$

Then $V(\cdot)$ is differentiable and locally positive definite. We prove that the derivative of $V(\cdot)$ along the trajectories of the system is nonpositive. Indeed, for almost all $t \geq 0$, we have

$$
\begin{aligned}
\dot{V}( & x(t))=\frac{d}{d t}(V \circ x(t)) \\
= & \frac{1}{2}\left\langle\frac{d}{d t}\left(M\left(x_{1}(t)\right)\right) x_{2}(t), x_{2}(t)\right\rangle+\left\langle x_{2}(t), M\left(x_{1}(t)\right) \dot{x}_{2}(t)\right\rangle+\left\langle\nabla \mathcal{V}\left(x_{1}(t)\right), \dot{x}_{1}(t)\right\rangle \\
= & \frac{1}{2}\left\langle\frac{d}{d t}\left(M\left(x_{1}(t)\right)\right) x_{2}(t), x_{2}(t)\right\rangle-\left\langle C\left(x_{1}(t), x_{2}(t)\right) x_{2}(t)+\nabla \mathcal{V}\left(x_{1}(t)\right)+F\left(x_{1}(t), x_{2}(t)\right)\right. \\
& \left.+\omega(t), x_{2}(t)\right\rangle+\left\langle\nabla \mathcal{V}\left(x_{1}(t)\right), x_{2}(t)\right\rangle \\
= & \left\langle\frac{1}{2}\left[\left\langle\frac{d}{d t}\left(M\left(x_{1}(t)\right)\right)-2 C\left(x_{1}(t), x_{2}(t)\right)\right] x_{2}(t), x_{2}(t)\right\rangle-\left\langle\omega(t)+F\left(x_{1}(t), x_{2}(t)\right), x_{2}(t)\right\rangle\right. \\
= & \left\langle\omega(t)+F\left(x_{1}(t), x_{2}(t)\right),-x_{2}(t)\right\rangle \leq \Phi(0)-\Phi\left(x_{2}(t)\right)+\alpha\left\|x_{2}(t)\right\| \leq 0
\end{aligned}
$$

for some $\omega(t) \in \partial \Phi\left(x_{2}(t)\right)$ and where Assumption 6.3(a) and the skew-symmetry property $\left(\mathcal{C}_{2}\right)$ of Assumption 3.2 have been used. Next, we prove that $x=0$ is stable. Since $V(\cdot)$ is locally positive definite, there exist $h>0$ and a strictly increasing function $\rho(\cdot) \in \mathcal{C}\left(\mathbb{R}^{+} ; \mathbb{R}\right)$ with $\rho(0)=0$ such that

$$
V(x) \geq \rho(\|x\|) \forall x \in \mathbb{B}_{h} .
$$

Without loss of generality, let $0<\varepsilon<h$ and let $c=\rho(\varepsilon)$. Because $V(\cdot)$ is locally positive definite, there exists $\eta>0$ such that $\mathbb{B}_{\eta} \subset \Omega_{c}^{\circ}=\left\{x \in \mathbb{R}^{2 n}: V(x)<c\right\}$. Let $\delta=\min \{\varepsilon, \eta\}$. Let $x_{0} \in \mathbb{B}_{\delta}$ and let $x\left(t ; x_{0}\right)$ be a solution of (6.4) satisfying the initial condition $x\left(0 ; x_{0}\right)=x_{0}$. Suppose that there exists $t_{1} \geq t_{0}$ such that $\left\|x\left(t_{1} ; x_{0}\right)\right\| \geq \varepsilon$. Since $x\left(\cdot ; x_{0}\right)$ is continuous, we may find some $t^{*}$ satisfying $\left\|x\left(t^{*} ; x_{0}\right)\right\|=\varepsilon$. Then

$$
V\left(x\left(t^{*} ; x_{0}\right)\right) \geq \rho\left(\left\|x\left(t^{*} ; x_{0}\right)\right\|\right)=\rho(\varepsilon) .
$$

On the other hand, $V(\cdot)$ is decreasing along the trajectory, and we have

$$
V\left(x\left(t^{*} ; x_{0}\right)\right) \leq V\left(x_{0}\right)<c=\rho(\varepsilon) .
$$

Our proof is finished by contradiction.

In the rest of this section, we will generalize the Krasovskii-LaSalle invariance principle to prove the asymptotic stability of the equilibrium set. First, we recall some definitions and properties. Let $x_{0} \in \mathbb{R}^{2 n}$ and $\psi\left(t ; x_{0}\right)$ be a solution of (6.4), denote the orbit of $\psi$ by

$$
\gamma(\psi):=\left\{\psi\left(t ; x_{0}\right): t \geq 0\right\} \subset \mathbb{R}^{2 n},
$$

and denote the limit set of $\psi$ by

$$
\Lambda(\psi):=\left\{p \in \mathbb{R}^{2 n}: \exists\left\{t_{i}\right\}, t_{i} \rightarrow \infty \text { as } i \rightarrow \infty \text { and } \psi\left(t_{i} ; x_{0}\right) \rightarrow p\right\} .
$$


A set $S \in \mathbb{R}^{2 n}$ is said to be weakly invariant if and only if for $x_{0} \in S$, there exists a solution of (6.4) starting at $x_{0}$ contained in $S$. It is said to be invariant if and only if for $x_{0} \in S$, all solutions of (6.4) starting at $x_{0}$ are contained in $S$.

Remark 6.2. The following properties are classical (see [2])

(i) If $\gamma(\psi)$ is bounded, then $\Lambda(\psi) \neq \emptyset$ and

$$
\lim _{t \rightarrow \infty} d\left(\psi\left(t ; x_{0}\right), \Lambda(\psi)\right)=0 .
$$

(ii) The set of stationary solutions $\mathcal{Y}$ is weakly invariant. In fact, if $x_{0} \in \mathcal{Y}$, then the solution $\psi\left(t ; x_{0}\right)=x_{0}, t \geq t_{0}$, is contained in $\mathcal{Y}$.

(iii) It is known that when $\mathcal{F}(\cdot)$ is upper semicontinuous with nonempty, convex, compact values and $\psi\left(t ; x_{0}\right)$ is a solution of $(6.4)$, then its limit set $\Lambda(\psi)$ is weakly invariant [9].

Lemma 6.4. The function $\mathcal{F}(\cdot)$ defined in (6.5) is upper semicontinuous with nonempty, convex, compact values.

Proof. The subdifferential $\partial \Phi(\cdot)$ is upper semicontinuous with nonempty, convex, compact values (Proposition 2.13). Therefore, particularly for all $x \in \mathbb{R}^{2 n}, \mathcal{F}(x)$ is nonempty, convex, and compact. It remains to prove that $\mathcal{F}(\cdot)$ is upper semicontinuous. Since the mapping

$$
x \rightarrow\left(\begin{array}{c}
x_{2} \\
M^{-1}\left(x_{1}\right)\left[\nabla \mathcal{V}\left(x_{1}\right)+C\left(x_{1}, x_{2}\right) x_{2}+F\left(x_{1}, x_{2}\right)\right]
\end{array}\right)
$$

is continuous, it is sufficient to prove that the mapping

$$
\mathcal{F}^{\prime}: x \rightarrow M^{-1}\left(x_{1}\right) \partial \Phi\left(x_{2}\right)
$$

is upper semicontinuous due to Proposition 2.10. Note that $\partial \Phi(\cdot)$ is also bounded on bounded sets, so $\mathcal{F}^{\prime}(\cdot)$ is bounded in a neighborhood of each point $x \in \mathbb{R}^{2 n}$. By Proposition 2.11, it is equivalent to prove that the graph of $\mathcal{F}^{\prime}(\cdot)$ is closed. Let $\left(y_{n}\right) \subset \mathbb{R}^{n}$ and $\left(x_{n}\right)=\left(\begin{array}{ll}x_{1 n}^{T} & x_{2 n}^{T}\end{array}\right)^{T} \subset \mathbb{R}^{2 n}$ be two sequences such that $y_{n} \rightarrow y$, $x_{n} \rightarrow x=\left(\begin{array}{ll}x_{1}^{T} & x_{2}^{T}\end{array}\right)^{T}$, and $y_{n} \in \mathcal{F}^{\prime}\left(x_{n}\right)=M^{-1}\left(x_{1 n}\right) \partial \Phi\left(x_{2 n}\right)$. Then for all $n \geq 1$, we have $M\left(x_{1 n}\right) y_{n} \in \partial \Phi\left(x_{2 n}\right)$. Since

$$
\begin{aligned}
\left\|M\left(x_{1 n}\right) y_{n}-M\left(x_{1}\right) y\right\| & \leq\left\|M\left(x_{1 n}\right) y_{n}-M\left(x_{1 n}\right) y\right\|+\left\|M\left(x_{1 n}\right) y-M\left(x_{1}\right) y\right\| \\
& \leq k_{2}\left\|y_{n}-y\right\|+k_{3}\left\|x_{1 n}-x_{1}\right\| .\|y\|,
\end{aligned}
$$

where $k_{2}, k_{3}$ are two constants defined in Assumption 3.1, we obtain that $M\left(x_{1 n}\right) y_{n} \rightarrow$ $M\left(x_{1}\right) y$. Moreover, $x_{2 n} \rightarrow x_{2}$ and the graph of $\partial \Phi(\cdot)$ is closed, and it results that $M\left(x_{1}\right) y \in \partial \Phi\left(x_{2}\right)$, i.e., $y \in M^{-1}\left(x_{1}\right) \partial \Phi\left(x_{2}\right)=\mathcal{F}^{\prime}(x)$. Therefore, the graph of $\mathcal{F}^{\prime}(\cdot)$ is closed and the result follows.

Lemma 6.5. Let the assumptions of Theorem 4.2 and Assumption 6.3(a) hold. Let $\Omega$ be a compact invariant subset of $\mathbb{R}^{2 n}$. Denote

$$
\mathcal{Z}_{\Omega}:=\left\{x=\left(x_{1}^{T} x_{2}^{T}\right)^{T} \in \Omega: \exists \omega \in \partial \Phi\left(x_{2}\right) \text { such that }\left\langle\omega+F\left(x_{1}, x_{2}\right), x_{2}\right\rangle=0\right\} .
$$

Let $\mathcal{M}$ be the largest weakly invariant set in the closure of $\mathcal{Z}_{\Omega}$. For each $x_{0} \in \Omega$, let $\psi\left(t ; x_{0}\right)$ be a solution of (6.4). Then, we have

$$
\lim _{t \rightarrow \infty} d\left(\psi\left(t ; x_{0}\right), \mathcal{M}\right)=0 .
$$

Proof. Since $x_{0} \in \Omega$ and $\Omega$ is invariant, we have $\gamma(\psi) \subset \Omega$. Then $\gamma(\psi)$ is bounded and 


$$
\lim _{t \rightarrow \infty} d\left(\psi\left(t ; x_{0}\right), \Lambda(\psi)\right)=0
$$

It is enough to prove that $\Lambda(\psi) \subset \overline{\mathcal{Z}}_{\Omega}$. Let us consider $V(\cdot)$ as in (6.9). Note that the function $V(\cdot)$ is $\mathcal{C}^{1}$; hence it is bounded on the compact set $\Omega$. Furthermore, since $V\left(\psi\left(t ; x_{0}\right)\right)$ is decreasing with respect to $t$, there exists a number $k$ such that $\lim _{t \rightarrow \infty} V\left(\psi\left(t ; x_{0}\right)\right)=k$. For each $p \in \Lambda(\psi)$, there exist $\left\{t_{i}\right\}, t_{i} \rightarrow \infty$ as $i \rightarrow \infty$ and $\psi\left(t_{i} ; x_{0}\right) \rightarrow p$. Then $V(p)=k$ due to the continuity of $V(\cdot)$. Hence $V(p)=k$ for all $p \in \Lambda(\psi)$. Let $z \in \Lambda(\psi)$. Since $\Lambda(\psi)$ is weakly invariant, there exists a solution $\phi(t ; z)$ of (6.4) lying in $\Lambda(\psi)$. Therefore,

$$
V(\phi(t ; z))=k
$$

for all $t \geq 0$, which implies

$$
0=\dot{V}(\phi(t ; z))=-\left\langle\omega(t)+F\left(\phi_{1}(t ; z), \phi_{2}(t ; z)\right), \phi_{2}(t ; z)\right\rangle
$$

for almost all $t \geq 0$, where $\omega(t) \in \partial \Phi\left(\phi_{2}(t ; z)\right)$ and $\phi(t)=\left(\phi_{1}^{T}(t) \phi_{2}^{T}(t)\right)^{T}$. Hence, we have

$$
\phi(t ; z) \in \mathcal{Z}_{\Omega}
$$

for almost all $t \geq 0$. Since $\phi\left(\cdot ; t_{0}, z\right)$ is continuous, we obtain

$$
z=\phi(0 ; z) \in \overline{\mathcal{Z}}_{\Omega}
$$

and the result follows.

Remark 6.3. Since $\mathcal{Z}_{\Omega} \subset \mathcal{Z}:=\left\{x=\left(x_{1}^{T} x_{2}^{T}\right)^{T} \in \mathbb{R}^{2 n}: \exists \omega \in \partial \Phi\left(x_{2}\right)\right.$ such that $\left.\left\langle x_{2}, \omega\right\rangle=0\right\}$, the conclusion is clearly true for $\mathcal{Z}$ instead of $\mathcal{Z}_{\Omega}$.

THEOREM 6.6 (attractivity). Let the assumptions of Theorem 4.2 hold. Furthermore, suppose the following:

(i) Assumption 6.3(b) holds.

(ii) $\mathcal{V}(\cdot)$ is radially unbounded. Then, for given $\psi_{0}=\left(q_{0}^{T} \dot{q}_{0}^{T}\right)^{T} \in \mathbb{R}^{2 n}$, if $\psi\left(t ; x_{0}\right)=$ $\left(q\left(t ; q_{0}, \dot{q}_{0}\right)^{T} \dot{q}\left(t ; q_{0}, \dot{q}_{0}\right)^{T}\right)^{T}$ is a solution of $(6.4)$, we have

$$
\lim _{t \rightarrow \infty} d\left(\psi\left(t ; x_{0}\right), \mathcal{Y}\right)=0
$$

or equivalently

$$
\lim _{t \rightarrow \infty} d\left(q\left(t ; q_{0}, \dot{q}_{0}\right), \mathcal{W}\right)=0 \text { and } \lim _{t \rightarrow \infty} \dot{q}\left(t ; q_{0}, \dot{q}_{0}\right)=0
$$

where $\mathcal{W}$ and $\mathcal{Y}$ are defined in (6.2) and (6.6), respectively.

Proof. Consider the same Lyapunov function as in Theorem 6.3; then

(1) $V \in \mathcal{C}^{1}\left(\mathbb{R}^{n}, \mathbb{R}\right)$,

(2) $V(\cdot)$ is bounded from below,

(3) $V(\cdot)$ is radially unbounded,

(4) $V(\cdot)$ is decreasing along the trajectories since the orbital derivative

$$
\begin{aligned}
\dot{V}(x(t)) & =\left\langle-x_{2}(t), \omega(t)+F\left(x_{1}(t), x_{2}(t)\right)\right\rangle \\
& \leq \Phi(0)-\Phi\left(x_{2}(t)\right)+\beta\left\|x_{2}(t)\right\| \leq-(\alpha-\beta)\left\|x_{2}(t)\right\| \leq 0,
\end{aligned}
$$

where $\omega(t) \in \partial \Phi\left(x_{2}(t)\right)$ due to assumption (i). We have $\dot{V}(x)=0$ if and only if $x_{2}=0$. 
For given $x_{0}=\left(q_{0}^{T} \dot{q}_{0}^{T}\right)^{T} \in \mathbb{R}^{2 n}$, let $\Omega=\left\{x \in \mathbb{R}^{2 n}: V(x) \leq V\left(x_{0}\right)\right\}$. Then $\Omega$ is a nonempty, compact subset of $\mathbb{R}^{2 n}$. Moreover, for $z \in \Omega$ and $\phi(t ; z)$ is a solution of (6.4), we have for all $t \geq 0, V(\phi(t ; z)) \leq V(\phi(0 ; z))=V(z) \leq$ $V\left(x_{0}\right)$, which implies $\phi(t ; z) \in \Omega$ for all $t \geq 0$. Hence $\Omega$ is invariant and $x_{0} \in \Omega$. Note that

$$
\begin{aligned}
\mathcal{Z}= & \left\{x=\left(x_{1}^{T} x_{2}^{T}\right)^{T} \in \mathbb{R}^{2 n}: \exists \omega \in \partial \Phi\left(x_{2}\right)\right. \text { such that } \\
& \left.\left\langle\omega+F\left(x_{1}, x_{2}\right), x_{2}\right\rangle=0\right\} \\
= & \mathbb{R}^{n} \times\{0\},
\end{aligned}
$$

where the last equality is obtained using assumption (i) of the theorem. In fact, we have $0=\left\langle\omega+F\left(x_{1}, x_{2}\right), x_{2}\right\rangle \geq \Phi\left(x_{2}\right)-\Phi(0)+\left\langle F\left(x_{1}, x_{2}\right), x_{2}\right\rangle \geq$ $(\alpha-\beta)\left\|x_{2}\right\| \geq 0$, which implies that $x_{2}=0$. Let $\mathcal{M}$ be the largest weakly invariant set in $\overline{\mathcal{Z}} \equiv \mathcal{Z}$. Then by Lemma 6.5 we have

$$
\lim _{t \rightarrow \infty} d\left(\psi\left(t ; x_{0}\right), \mathcal{M}\right)=0 .
$$

We recall that the set $\mathcal{Y}$ of stationary solutions of (6.4) is weakly invariant and $\mathcal{Y}=\mathcal{W} \times\{0\}$. Hence, $\mathcal{Y}$ is a weakly invariant subset of $\mathcal{Z}$. We now prove that $\mathcal{Y} \equiv \mathcal{M}$. Indeed, let $\mathcal{D}$ be a weakly invariant set in $\mathcal{Z}$ and take $z=\left(\begin{array}{ll}z_{1}^{T} & z_{2}^{T}\end{array}\right)^{T} \in \mathcal{D}$. Then there exists a solution $\theta(t ;, z)$ lying in $D$ and for almost all $t \geq 0$ :

$$
\left\{\begin{array}{l}
\dot{\theta}_{1}(t ; z)=\theta_{2}(t ; z) \\
\dot{\theta}_{2}(t ; z)+M^{-1}\left(\theta_{1}(t ; z)\right)\left[C\left(\theta_{1}(t ; z), \theta_{2}(t ; z)\right) \theta_{2}(t ; z)+\nabla \mathcal{V}\left(\theta_{1}(t ; z)\right)\right. \\
\left.+F\left(\theta_{1}(t ; z), \theta_{2}(t ; z)\right)\right] \in-M^{-1}\left(\theta_{1}(t ; z)\right) \partial \Phi\left(\theta_{2}(t ; z)\right)
\end{array}\right.
$$

Since the orbit $\gamma(\theta) \subset D \subset \mathcal{Z}$, we infer from (6.14) that $\theta_{2}(t ; z)=0$ for all $t \geq 0$. Hence, $z_{2}=0, \theta_{1}(t ; z) \equiv \theta_{1}(0 ; z)=z_{1}$ and $\mathcal{V}\left(z_{1}\right)+F\left(z_{1}, 0\right) \in-\partial \Phi(0)$. This means that $z \in \mathcal{Y}$, which leads to $\mathcal{D} \subset \mathcal{Y}$. Therefore, $\mathcal{Y}$ is the largest weakly invariant set in $\mathcal{Z}$ and

$$
\lim _{t \rightarrow \infty} d\left(\psi\left(t ; x_{0}\right), \mathcal{Y}\right)=0,
$$

or equivalently

$$
\lim _{t \rightarrow \infty} d\left(q\left(t ; q_{0}, \dot{q}_{0}\right), \mathcal{W}\right)=0 \text { and } \lim _{t \rightarrow \infty} \dot{q}\left(t ; q_{0}, \dot{q}_{0}\right)=0 .
$$

7. Finite-time convergence. In this section, the finite-time convergence of trajectories toward the equilibrium point is investigated. This property is, besides the robustness, a crucial feature of discontinuous and sliding mode controllers systems $[40,44,47]$.

Theorem 7.1. Let Assumptions 3.1, 3.2, 3.3, 6.1, and 6.3(b) hold and let $(q(\cdot), \dot{q}(\cdot))$ be a solution of the system (1.1). The energy function is given by $V(q, \dot{q})=$ $\frac{1}{2}\langle M(q) \dot{q}, \dot{q}\rangle+\mathcal{V}(q)$. Suppose that $\mathcal{V}(\cdot)$ is radially unbounded. Then

(i) $\dot{q} \in L^{\infty}\left([0,+\infty), \mathbb{R}^{n}\right)$;

(ii) $\dot{q} \in L^{1}\left([0,+\infty), \mathbb{R}^{n}\right)$ and hence $q_{\infty}=\lim _{t \rightarrow \infty} q(t)$ exists, and furthermore, we have $\lim _{t \rightarrow \infty} \dot{q}(t)=0$;

(iii) the limit point $q_{\infty}$ satisfies

$$
\nabla \mathcal{V}\left(q_{\infty}\right)+F\left(q_{\infty}, 0\right) \in-\partial \Phi(0),
$$

i.e., $q_{\infty}$ is an equilibrium point of (1.1). 
Proof.

(i) Differentiating $V(\cdot)$ along the system's trajectories as in the proof of Theorem 6.3 , we have for a.e. $t \geq 0$,

$$
\dot{V}(q(t), \dot{q}(t)) \leq \Phi(0)-\Phi(\dot{q}(t))+\beta\|\dot{q}(t)\| \leq-(\alpha-\beta)\|\dot{q}(t)\| \leq 0 .
$$

We have $\frac{1}{2} k_{1}\|\dot{q}(t)\|^{2} \leq V(q(t), \dot{q}(t))-\mathcal{V}(q(t)) \leq V\left(q_{0}, \dot{q}_{0}\right)-\inf \mathcal{V}$. So $\dot{q} \in$ $L^{\infty}\left([0,+\infty), \mathbb{R}^{n}\right)$

(ii) Next, we integrate inequality (7.2) between 0 and $t$, which yields

$$
(\alpha-\beta) \int_{0}^{t}\|\dot{q}(s)\| d s \leq V\left(q_{0}, \dot{q}_{0}\right)-V(q(t), \dot{q}(t)) \leq V\left(q_{0}, \dot{q}_{0}\right)-\inf \mathcal{V}
$$

Let $t \rightarrow+\infty$; it follows that $\dot{q} \in L^{1}\left([0,+\infty), \mathbb{R}^{n}\right)$.

From $\mathcal{V}(q(t)) \leq V(q(t), \dot{q}(t)) \leq V\left(q_{0}, \dot{q}_{0}\right)<\infty$, and the radial unboundedness of $\mathcal{V}(\cdot)$, we have $q \in L^{\infty}\left([0,+\infty), \mathbb{R}^{n}\right)$. Then $\nabla \mathcal{V}(q)$ is bounded due to the boundedness of $\nabla \mathcal{V}(\cdot)$ on bounded sets (see Assumption 3.3). Since the map $\dot{q}(\cdot)$ is bounded and $\partial \Phi(\cdot)$ is bounded on bounded sets (see Proposition 2.13), we have that $\partial \Phi(\dot{q})$ is bounded. Furthermore, from $\left(\mathcal{C}_{1}\right)$ of Assumption 3.2, we have $\|C(q, \dot{q}) \dot{q}\| \leq k_{4}\|\dot{q}\|^{2}$, which implies that $C(q, \dot{q}) \dot{q}$ is also bounded. Note that $F(\cdot, q, \dot{q})$ is also bounded. Therefore, $\ddot{q}$ is bounded and hence $\dot{q}$ is Lipschitz continuous. This combined with the fact that $\dot{q} \in L^{1}\left([0,+\infty), \mathbb{R}^{n}\right)$ classically implies that $\lim _{t \rightarrow \infty} \dot{q}(t)=0$, see, e.g., [17, section 4.3].

(iii) Assume that $\nabla \mathcal{V}\left(q_{\infty}\right)+F\left(q_{\infty}, 0\right) \notin-\partial \Phi(0)$ or, equivalently, $0 \notin \Sigma_{0}:=\{z \in$ $\left.\mathbb{R}^{n}: z \in \partial \Phi(0)+\nabla \mathcal{V}\left(q_{\infty}\right)+F\left(q_{\infty}, 0\right)\right\}$. Then, the convex compact set $\{0\}$ and the closed convex set $\Sigma_{0}$ can be separated strictly by a hyperplane (see, for instance, Corollary 11.4.2, in [37]). It means that there exist $v \in \mathbb{R}^{n}$ and $k>0$ such that

$$
\left\langle p+\nabla \mathcal{V}\left(q_{\infty}\right)+F\left(q_{\infty}, 0\right), v\right\rangle>k
$$

for all $p \in \partial \Phi(0)$. Then there exists $t^{\prime} \geq 0$ such that

$$
\left\langle\dot{q}^{*}(t)+\nabla \mathcal{V}(q(t))+F(q(t), \dot{q}(t)), v\right\rangle>k
$$

for all $t \geq t^{\prime}, \dot{q}^{*}(t) \in \partial \Phi(\dot{q}(t))$. Indeed, suppose the contrary; then there exist sequences $\left(w_{n}\right),\left(t_{n}\right)$ such that $t_{n} \rightarrow+\infty, w_{n} \in \partial \Phi\left(\dot{q}\left(t_{n}\right)\right)$ and

$$
\left\langle w_{n}+\nabla \mathcal{V}\left(q\left(t_{n}\right)\right)+F\left(q\left(t_{n}\right), \dot{q}\left(t_{n}\right)\right), v\right\rangle \leq k
$$

for all $n \geq 1$. The sequence $\left(w_{n}\right)$ is bounded since the set-valued mapping $\partial \Phi(\cdot)$ is bounded on bounded sets. Therefore, there exists $w \in \mathbb{R}^{n}$ and a subsequence of $\left(w_{n}\right)$, still denoted by $\left(w_{n}\right)$ such that $w_{n} \rightarrow w$. Furthermore, $\dot{q}\left(t_{n}\right) \rightarrow 0$ and the graph of $\partial \Phi(\cdot)$ is closed in $\mathbb{R}^{n} \times \mathbb{R}^{n}$; we obtain that $w \in \partial \Phi(0)$. In addition to the assumptions on $\nabla \mathcal{V}(\cdot)$ and $F(\cdot)$, we have

$$
\left\langle w+\nabla \mathcal{V}\left(q_{\infty}\right)+F\left(q_{\infty}, 0\right), v\right\rangle \leq k
$$

which is a contradiction to (7.3).

From (7.4), we have

$$
\begin{aligned}
& \langle-M(q(t)) \ddot{q}(t)-C(q(t), \dot{q}(t)) \dot{q}(t), v\rangle>k \forall t \geq t^{\prime} \\
& \Rightarrow k\left(t-t^{\prime}\right)<\int_{t^{\prime}}^{t}\langle-M(q(s)) \ddot{q}(s)-C(q(s), \dot{q}(s)) \dot{q}(s), v\rangle d s .
\end{aligned}
$$


Note that

$$
\begin{aligned}
& \frac{d}{d t}(M(q(t)) \dot{q}(t))=M(q(t)) \ddot{q}(t)+\frac{d}{d t}(M(q(t))) \dot{q}(t) \\
& \quad=M(q(t)) \ddot{q}(t)+\left[C(q(t), \dot{q}(t))+C(q(t), \dot{q}(t))^{T}\right] \dot{q}(t) \\
& \quad \Rightarrow M(q(t)) \ddot{q}(t)+C(q(t), \dot{q}(t)) \dot{q}(t)=\frac{d}{d t}(M(q(t)) \dot{q}(t))-C(q(t), \dot{q}(t))^{T} \dot{q}(t),
\end{aligned}
$$

where we used $\left(\mathcal{C}_{2}\right)$ of Assumption 3.2. Therefore, we have

$$
\begin{aligned}
& \left|\int_{t^{\prime}}^{t}\langle-M(q(s)) \ddot{q}(s)-C(q(s), \dot{q}(s)) \dot{q}(s), v\rangle d s\right| \\
& \quad=\left|\left\langle M\left(q\left(t^{\prime}\right)\right) \dot{q}\left(t^{\prime}\right)-M(q(t)) \dot{q}(t), v\right\rangle+\int_{t^{\prime}}^{t}\left\langle C(q(s), \dot{q}(s))^{T} \dot{q}(s), v\right\rangle d s\right| \\
& \quad \leq\left\|M\left(q\left(t^{\prime}\right)\right)\right\|_{m}\left\|\dot{q}\left(t^{\prime}\right)\right\|\|v\|+\|M(q(t))\|_{m}\|\dot{q}(t)\|\|v\|+k_{4}\|v\| \int_{t^{\prime}}^{t}\|\dot{q}(s)\|^{2} d s \\
& \quad \leq k_{2}\left\|\dot{q}\left(t^{\prime}\right)\right\|\|v\|+k_{2}\|\dot{q}(t)\|\|v\|+k_{7} \int_{t^{\prime}}^{t}\|\dot{q}(s)\| d s<K,
\end{aligned}
$$

where $k_{7}, K$ are constants, due to the facts that $\dot{q} \in L^{\infty}\left([0,+\infty), \mathbb{R}^{n}\right)$ and $\dot{q} \in L^{1}\left([0,+\infty), \mathbb{R}^{n}\right)$. Then, using (7.6), we obtain for all $t \geq t^{\prime}$

$$
k\left(t-t^{\prime}\right) \leq K
$$

which is a contradiction. Hence, we have $\nabla \mathcal{V}\left(q_{\infty}\right)+F\left(q_{\infty}, 0\right) \in-\partial \Phi(0)$.

In the following theorem, we give a sufficient condition ensuring that each trajectory of the system converges in finite time to an equilibrium point solution of (7.1).

TheOREM 7.2 (finite-time convergence). Let the assumptions of Theorem 7.1 hold. Moreover, assume that $-\nabla \mathcal{V}\left(q_{\infty}\right)-F\left(q_{\infty}, 0\right) \notin \mathrm{bd}(\partial \Phi(0))$. Then there exists $t_{f}<\infty\left(t_{f} \geq 0\right)$ such that $q(t)=q_{\infty}$ for every $t \geq t_{f}$.

Proof. From $-\nabla \mathcal{V}\left(q_{\infty}\right)-F\left(q_{\infty}, 0\right) \in \partial \Phi(0)$ and $-\nabla \mathcal{V}\left(q_{\infty}\right)-F\left(q_{\infty}, 0\right) \notin \mathrm{bd}(\partial \Phi(0))$, we have $-\nabla \mathcal{V}\left(q_{\infty}\right)-F\left(q_{\infty}, 0\right) \in \operatorname{int}(\partial \Phi(0))$. Let $2 \varepsilon=d\left(-\nabla \mathcal{V}\left(q_{\infty}\right)-F\left(q_{\infty}, 0\right)\right.$, $\operatorname{bd}(\partial \Phi(0)))$. Combining with $\lim _{t \rightarrow+\infty}\{\nabla \mathcal{V}(q(t))+F(q(t), \dot{q}(t))\}=\nabla \mathcal{V}\left(q_{\infty}\right)+F\left(q_{\infty}, 0\right)$, there exists a fixed $t_{1} \geq t_{0}$ such that for every $t \geq t_{1}$, we have

$$
-\nabla \mathcal{V}(q(t))-F(q(t), \dot{q}(t)) \in \mathbb{B}_{\epsilon}\left(-\nabla \mathcal{V}\left(q_{\infty}\right)-F\left(q_{\infty}, 0\right)\right)
$$

and hence

$$
-\nabla \mathcal{V}(q(t))-F(q(t), \dot{q}(t))+\mathbb{B}_{\epsilon} \in \partial \Phi(0) .
$$

This means that for all $t \geq t_{1}$ and for all $u \in \mathbb{B}_{\epsilon}$, we have equivalently (see Definition 2.12)

$$
\Phi(\dot{q}(t)) \geq\langle-\nabla \mathcal{V}(q(t))-F(q(t), \dot{q}(t))+u, \dot{q}(t)\rangle,
$$

which implies

$$
\Phi(\dot{q}(t)) \geq\langle-\nabla \mathcal{V}(q(t))-F(q(t), \dot{q}(t)), \dot{q}(t)\rangle+\epsilon\|\dot{q}(t)\| \forall t \geq t_{1} .
$$

We have $\dot{V}(q(t), \dot{q}(t)) \leq-\Phi(\dot{q}(t))-\langle F(q(t), \dot{q}(t)), \dot{q}(t)\rangle$ for almost all $t \geq t_{0}$. (See the proof of Theorem 6.3.) Therefore

$$
\frac{1}{2} \frac{d}{d t}\langle M(q(t)) \dot{q}(t), \dot{q}(t)\rangle+\langle\nabla \mathcal{V}(q(t)), \dot{q}(t)\rangle+\langle F(q(t), \dot{q}(t)), \dot{q}(t)\rangle+\Phi(\dot{q}(t)) \leq 0,
$$

and hence for almost all $t \geq t_{1}$, 


$$
\frac{d}{d t}\langle M(q(t)) \dot{q}(t), \dot{q}(t)\rangle+2 \epsilon\|\dot{q}(t)\| \leq 0
$$

Let $c(t):=\langle M(q(t)) \dot{q}(t), \dot{q}(t)\rangle \leq k_{2}\|\dot{q}(t)\|^{2} \Rightarrow\|\dot{q}(t)\| \geq \sqrt{\frac{c(t)}{k_{2}}}$, where $k_{2}$ is in $\left(\mathcal{M}_{1}\right)$ of Assumption 3.1. Therefore

$$
\dot{c}(t)+\alpha \sqrt{c(t)} \leq 0 \text { for a.e. } t \geq t_{1},
$$

where $\alpha:=2 \epsilon / \sqrt{k_{2}}$. Assume that for every $t \geq t_{1}, c(t)>0$. Dividing (7.8) by $\sqrt{c(t)}$ and integrating on $\left[t_{1}, t\right]$, we have

$$
\sqrt{c(t)}-\sqrt{c\left(t_{1}\right)} \leq-\alpha\left(t-t_{1}\right) \forall t \geq t_{1},
$$

which is a contradiction. So there exists $t_{f} \geq t_{1}$ such that $c\left(t_{f}\right)=0$. Note that $\dot{c}(t) \leq 0$ for almost all $t \geq t_{1}$, so $c(t) \leq c\left(t_{f}\right)$ for all $t \geq t_{f}$, which implies $c(t)=0$ for all $t \geq t_{f}$. Therefore, $\dot{q}(t)=0$ for all $t \geq t_{f}$, i.e., $q(t)=q_{\infty}$ for every $t \geq t_{f}$.

Proposition 7.3. Let assumptions of Theorem 7.2 hold. Let $t_{f}$ to be the first time instant such that $\dot{q}(t)=0, q(t)=q_{\infty}$ for every $t \geq t_{f}$ and $t_{1}$ be the first time instant such that $-\nabla \mathcal{V}(q(t))-F(q(t), \dot{q}(t))$ lies in the ball $\mathbb{B}_{\varepsilon}\left(-\nabla \mathcal{V}\left(q_{\infty}\right)-F\left(q_{\infty}, 0\right)\right)$ and stays there for all $t \geq t_{1}$. Then

$$
t_{f} \leq t_{1}+\frac{\sqrt{k_{2}} \sqrt{c\left(t_{1}\right)}}{d\left(-\nabla \mathcal{V}\left(q_{\infty}\right)-F\left(q_{\infty}, 0\right), \operatorname{bd}(\partial \Phi(0))\right)} .
$$

Proof. If $c\left(t_{1}\right)=0$, the proof is trivial since $t_{f}=t_{1}$. If $c\left(t_{1}\right)>0$, we must have $c(t)>0$ for all $t \in\left[t_{1}, t_{f}\right)$ and $c\left(t_{f}\right)=0$. From (7.9) we have

$$
-\sqrt{c\left(t_{1}\right)}=\sqrt{c\left(t_{f}\right)}-\sqrt{c\left(t_{1}\right)} \leq-\alpha\left(t_{f}-t_{1}\right)
$$

which implies

$$
t_{f} \leq t_{1}+\frac{\sqrt{c\left(t_{1}\right)}}{\alpha}=t_{1}+\frac{\sqrt{k_{2}} \sqrt{c\left(t_{1}\right)}}{d\left(-\nabla \mathcal{V}\left(q_{\infty}\right)-F\left(q_{\infty}, 0\right), \operatorname{bd}(\partial \Phi(0))\right)} .
$$

The distance function in the denominator in the right-hand side of (7.10) depends on the control functions and consequently on the control gains. It may therefore be tuned to adjust the settling time $t_{f}$, a property desirable in practice. Before $t_{1}$, it is possible to have some time instances at which $\dot{q}(\cdot)$ is zero. But we can prove that these points must be isolated with additional assumptions.

Proposition 7.4. Let the assumptions of Theorem 7.2 hold. Suppose that $t^{\prime}<t_{f}$ satisfies $\dot{q}\left(t^{\prime}\right)=0$, where $t_{f}$ is defined in Proposition 7.3. Then $-\mathcal{V}\left(q\left(t^{\prime}\right)\right)-$ $F\left(q\left(t^{\prime}\right), 0\right) \notin \operatorname{int}(\partial \Phi(0))$. If $-\mathcal{V}\left(q\left(t^{\prime}\right)\right)-F\left(q\left(t^{\prime}\right), 0\right) \notin \operatorname{bd}(\partial \Phi(0))$, then $t^{\prime}$ is an isolated point in the set $\left\{s \in \mathbb{R}^{+}: \dot{q}(s)=0\right\}$.

Proof. Suppose that $-\mathcal{V}\left(q\left(t^{\prime}\right)\right)-F\left(q\left(t^{\prime}\right), 0\right) \in \operatorname{int}(\partial \Phi(0))$. Similarly as in the proof of Theorem 7.2, due to the continuity of $\mathcal{V} \circ q(\cdot)$ and $F(q(\cdot), \dot{q}(\cdot))$, there exist $\delta>0, \varepsilon>0$ such that

$$
-\nabla \mathcal{V}(q(t))-F(q(t), \dot{q}(t))+\mathbb{B}_{\epsilon} \in \partial \Phi(0)
$$

for all $t \in\left(t^{\prime}-\delta, t^{\prime}+\delta\right)$. Then we also have

$$
\dot{c}(t)+\alpha \sqrt{c(t)} \leq 0 \text { for a.e. } t \in\left(t^{\prime}-\delta, t^{\prime}+\delta\right),
$$


where $c(t)$ and $\alpha$ are defined in the proof of Theorem 7.2. Then, $c(t) \leq c\left(t^{\prime}\right)=0$ for all $t \in\left[t^{\prime}, t^{\prime}+\delta\right)$. Hence, for all $t \in\left[t^{\prime}, t^{\prime}+\delta\right)$, we have $\dot{q}(t)=0$ and $q(t)=q\left(t^{\prime}\right)$. Let $t_{\max }=\sup \left\{\tau>t^{\prime}: \dot{q}(t)=0\right.$ for all $\left.t \in\left[t^{\prime}, \tau\right)\right\}$. Assume that $t_{\max }<+\infty$. Since $q(\cdot), \dot{q}(\cdot)$ are continuous, we have $\dot{q}\left(t_{\max }\right)=0$ and $q\left(t_{\max }\right)=q\left(t^{\prime}\right)$. Repeating the argument above for $t_{\max }$ instead of $t^{\prime}$, we obtain a contradiction with the definition of $t_{\max }$. So, we must have $t_{\max }=+\infty$ but it is impossible since $t^{\prime}<t_{f}$. Therefore, $-\mathcal{V}\left(q\left(t^{\prime}\right)\right)-F\left(q\left(t^{\prime}\right), 0\right) \notin \operatorname{int}(\partial \Phi(0))$.

If $-\mathcal{V}\left(q\left(t^{\prime}\right)\right)-F\left(q\left(t^{\prime}\right), 0\right) \notin \operatorname{bd}(\partial \Phi(0))$, we have $-\mathcal{V}\left(q\left(t^{\prime}\right)\right)-F\left(q\left(t^{\prime}\right), 0\right) \notin \partial \Phi(0)$. Using a separation theorem [37], there exist $v \in \mathbb{R}^{n}$ and $k>0$ satisfying

$$
\left\langle\mathcal{V}\left(q\left(t^{\prime}\right)\right)+F\left(q\left(t^{\prime}\right), 0\right)+p, v\right\rangle>k
$$

for all $p \in \partial \Phi(0)$. Similarly as in the proof of (iii) of Theorem 7.1, we can find $\sigma>0$ such that

$$
\left\langle\mathcal{V}(q(t))+F(q(t), \dot{q}(t))+\dot{q}^{*}(t), v\right\rangle>k
$$

for all $t \in\left(t^{\prime}-\sigma, t^{\prime}+\sigma\right), \dot{q}^{*}(t) \in \partial \Phi(\dot{q}(t))$ due to the continuity of $\mathcal{V} \circ q$ and the graph-closedness property of $\partial \Phi$. This implies that for all $t \in\left(t^{\prime}-\sigma, t^{\prime}+\sigma\right)$,

$$
\langle-M(q(t)) \ddot{q}(t)-C(q(t), \dot{q}(t)) \dot{q}(t), v\rangle>k .
$$

If there exists $0<\delta<\sigma$ such that $\dot{q}(t)=0$ for all $t \in\left(t^{\prime}-\delta, t^{\prime}+\delta\right)$, then $\ddot{q}(t)=0$ for all $t \in\left(t^{\prime}-\delta, t^{\prime}+\delta\right)$ and

$$
0=\langle-M(q(t)) \ddot{q}(t)+C(q(t), \dot{q}(t)) \dot{q}(t), v\rangle>k>0
$$

for all $t \in\left(t^{\prime}-\delta, t^{\prime}+\delta\right)$, a contradiction. Hence, $t^{\prime}$ is isolated in the zero set of $\dot{q}(\cdot)$.

Proposition 7.5. Assume that for a given initial condition, system (1.1) has a unique solution $(q(\cdot), \dot{q}(\cdot))$. Let assumptions of Theorem 7.2 hold. Then, the set $D=\left\{t<t_{f}: \dot{q}(t)=0\right\}$ is discrete and countable.

Proof. If $D=\emptyset$, the conclusion is trivial. Otherwise, let $t^{\prime} \in D$. If $-\mathcal{V}\left(q\left(t^{\prime}\right)\right)-$ $F\left(q\left(t^{\prime}\right), 0\right) \in \partial \Phi(0)$, we must have $q(t)=q\left(t^{\prime}\right)$ for all $t \geq t^{\prime}$, due to the uniqueness of solutions. Hence $t^{\prime} \geq t_{f}$, a contradiction with $t^{\prime} \in D$. Therefore $-\mathcal{V}\left(q\left(t^{\prime}\right)\right)-$ $F\left(q\left(t^{\prime}\right), 0\right) \notin \partial \Phi(0)$. Similarly as in the proof of Proposition 7.4, we obtain that $t^{\prime}$ is isolated in $D$. Since it is true for each $t^{\prime} \in D$, we conclude that $D$ is discrete and countable.

8. Conclusion. In this paper, the well-posedness of nonlinear Lagrangian dynamical systems with a discontinuous controller is analyzed. An existence result is proved by using the Moreau-Yosida regularization. Some conditions ensuring the uniqueness of the trajectory are given. We also study the Lyapunov stability as well as the attractivity properties of the set of stationary solutions of the Lagrangian dynamical systems. We conclude the paper by giving sufficient conditions ensuring finite-time convergence of the trajectory to an equilibrium point with an estimation of the settling time. The paper raises some important questions about the wellposedness, the robustness, and the stability analysis of a set-valued controller for the Lagrangian dynamical systems. This problem is known to be difficult and there are few results in that direction. Our methodology is original and uses tools from convex and set-valued analysis. It will be interesting to incorporate the proposed theory in some practical and concrete situation in engineering and to study the discrete-time version of the discontinuous controllers studied in this paper. This is out of the scope of the current paper and likely will be the subject of a future work. 


\section{REFERENCES}

[1] S. Adly, H. Aтtouch, And A. Савот, Finite time stabilization of nonlinear oscillators subject to dry friction, in Progresses in Nonsmooth Mechanics and Analysis (P. Alart, O. Maisonneuve, and R.T. Rockafellar, eds.), Advances in Mathematics and Mechanics, Kluwer, Dordrecht, Netherlands, 2006, pp. 289-304.

[2] S. Adly AND D. Goeleven, A stability theory for second-order nonsmooth dynamical systems with application to friction problems, J. Math. Pures Appl., 83 (2004), pp. 17-51.

[3] V. Acary And B. Brogliato, Implicit Euler numerical scheme and chattering-free implementation of sliding mode systems, Systems Control Lett., 59 (2010), pp. 284-293.

[4] V. Acary, B. Brogliato, and Y. Orlov, Chattering-free digital sliding-mode control with state observer and disturbance rejection, IEEE Trans. Automat. Control, 57 (2012), pp. 1087-1101.

[5] J. C. Alexander And T. I. Seidman, Sliding modes in intersecting switching surfaces, I: Blending, Houston J. Math., 24 (1998), pp. 545-569.

[6] J. C. Alexander And T. I. Seidman, Sliding modes in intersecting switching surfaces, II: Hysteresis, Houston J. Math., 25 (1999), pp. 185-211.

[7] S. Arimoto, Control Theory of Nonlinear Mechanical Systems: A Passivity-Based and Circuittheoretic Approach, Oxford University Press, New York, 1996.

[8] J. P. Aubin And A. Cellina, Differential Inclusions, Grundlehren Math. Wisse. 264, SpringerVerlag, Berlin, 1984.

[9] A. Bacciotti And F. Ceragioli, Stability and stabilization of discontinuous systems and nonsmooth Lyapunov functions, ESAIM Control Optim. Calc. Var., 4 (1999), pp. 361-376.

[10] G. Bartolini, L. Fridman, A. Pisano, and E. Usai eds., Modern Sliding Mode Control Theory New Perspectives and Applications, Lecture Notes in Control and Inform. Sci. 375, Springer-Verlag, Berlin, 2008.

[11] D. P. Bertsekas, A. Nedic, And A. E. Ozdaglaz, Convex Analysis and Optimization, Athena Scientific, Belmont, MA, 2003.

[12] S. P. Bhat And D. S. Bernstein, Finite time stability of continuous autonomous systems, SIAM J. Control Optim., 38 (2000), pp. 751-766.

[13] H. Brezis, Opérateurs Maximaux Monotones et Semi-groupes de Contractions dans les Espaces de Hilbert, Math. Stud. 5, American Elsevier, New York, 1973.

[14] B. Brogliato, Absolute stability and the Lagrange-Dirichlet theorem with monotone multivalued mappings, Systems Control Lett., 51 (2004), pp. 343-353.

[15] B. Brogliato and D. Goeleven, The Krasovskii-LaSalle invariance principle for a class of unilateral dynamical systems, Math. Control Signals Systems, 17 (2005), pp. 57-76.

[16] B. Brogliato and D. Goeleven, Well-posedness, stability and invariance results for a class of multivalued Lur'e dynamical systems, Nonlinear Anal., 74 (2011), pp. 195-212.

[17] B. Brogliato, R. Lozano, B. Maschke, and O. Egeland, Dissipative Systems Analysis and Control, 2nd ed., Springer, London, 2007.

[18] A. Савот, Stabilization of oscillators subject to dry friction: finite time convergence versus exponential decay results, Trans. Amer. Math. Soc., 360 (2008), pp. 103-121.

[19] Y. FENG, X. YU, AND Z. MAN, Non-singular terminal sliding mode control of rigid manipulators, Automatica, 38 (2002), pp. 2159-2167.

[20] A. F. Filippov, Differential Equations with Discontinuous Righthand Sides, Kluwer Academic, Norwell, MA, 1988.

[21] L. Fridman, J. Moreno, and R. Iriarte, eds., Sliding Modes After the First Decade of the 21 st Century. State of the Art, Lecture Notes in Control and Inform. Sci. 412, SpringerVerlag, 2012.

[22] Z. Galias And X. YU, Complex discretization behaviours of a simple sliding-mode control system, IEEE Trans. Circuits Systems II Express Briefs, 53 (2006), pp. 652-656.

[23] Z. Galias And X. YU, Analysis of zero-order holder discretization of two-dimensional slidingmode control systems, IEEE Trans. Circuits Systems II Express Briefs, 55 (2008), pp. 12691273.

[24] D. Goeleven, D. Motreanu, and V. Motreanu, On the stability of stationary solutions of first-order evolution variational inequalities, Adv. Nonlinear Var. Inequal., 6 (2003), pp. 1-30.

[25] Y. Hong, Y. Xu, And J. Huang, Finite-time control for robot manipulators, Systems Control Lett., 46 (2002), pp. 243-253.

[26] O. Kaynak, A. Bartoszewicz, and V.I. Utkin, eds., IEEE Trans. Industrial Electronics, 55 (2008).

[27] M. Johansson, Piecewise Linear Control Systems, Lecture Notes in Comput. and Inform. Sci. 284, Springer, London, 2003. 
[28] O. Kaynak, A. Bartoszewicz, and V.I. Utkin, eds., IEEE Trans. Industrial Electronic, 56 (2009).

[29] R. I. Leine And N. Wouw, Stability and Convergence of Mechanical Systems with Unilateral Constraints, Springer-Verlag, Berlin, 2008.

[30] A. M. Michel And L. Hou, Relaxation of hypotheses in LaSalle-Krasovskii-type invariance results, SIAM J. Control Optim., 49 (2011), pp. 1383-1403.

[31] E. Moulay and W. Perruquetti, Finite time stability and stability and stabilization of a class of continuous systems, J. Math. Anal. Appl., 323 (2006), pp. 1430-1443.

[32] E. Moulay and W. Perruquetti, Finite time stability of differential inclusions, IMA J. Math. Control Inform., 22 (2005), pp. 465-475.

[33] Y. OrLov, Discontinuous Systems, Springer, London, 2009.

[34] Y. ORLOv, Finite time stability and robust control synthesis of uncertain switched systems, SIAM J. Control Optim., 43 (2004), pp. 1253-1271.

[35] L. Paoli and M. Schatzman, Mouvement à nombre fini de degres de liberteé avec contraintes unilatérales. Cas avec perte d'énergie, Modelisation Mathématique et Analyse Numérique, 27, (1993), pp. 673-717.

[36] R. R. Phelps, Convex Functions, Monotone Operators and Differentiability, 2nd ed., Lecture Notes in Math. 1364, Springer-Verlag, Berlin, 1993.

[37] R. T. Rockafellar, Convex Analysis, Princeton Landmarks Math., Princeton, NJ, 1970.

[38] E. P. RYAN, An integral invariance principle for differential inclusions with applications in adaptive control, SIAM J. Control Optim., 36 (1998), pp. 960-980.

[39] M. Schatzman, A class of nonlinear differential equations of second order in time, Nonlinear Analysis, 2 (1978), pp. 355-373.

[40] J. J. E. Slotine, The robust control of robot manipulators, Int. J. Robotics Research, 4 (1985), pp. 49-64.

[41] M. W. Spong, S. Hutchinson, And M. Vidyasagar, Robot Modeling and Control, Jhon Wiley, New York, 2005.

[42] J. B. H. Urruty and C. Lemaréchal, Fundamentals of Convex Analysis, Springer-Verlag, Berlin, 2001.

[43] V. UtKin, Variable structure systems with sliding mode, IEEE Trans. Automat. Control, 22 (1977), pp. 212-222.

[44] V. Utkin, Sliding Modes in Control Optimization, Springer-Verlag, Berlin, 1992.

[45] V. Utkin, J. Guldner, And J. Shi, Sliding Mode Control in Electro-Mechanical Systems, 2nd ed., CRC Press, Boca Raton, FL, 2009.

[46] K. D. Young, V. I. Utkin, And U. Ozguner, A control engineer's guide to sliding mode control, IEEE Trans. Control Systems Technology, 7 (1999), pp. 328-342.

[47] S. Yu, X. Yu, B. Shirinzadeh, And Z. Man, Continuous finite-time control for robotic manipulators with terminal sliding mode, Automatica, 41 (2005), pp. 1957-1964. 